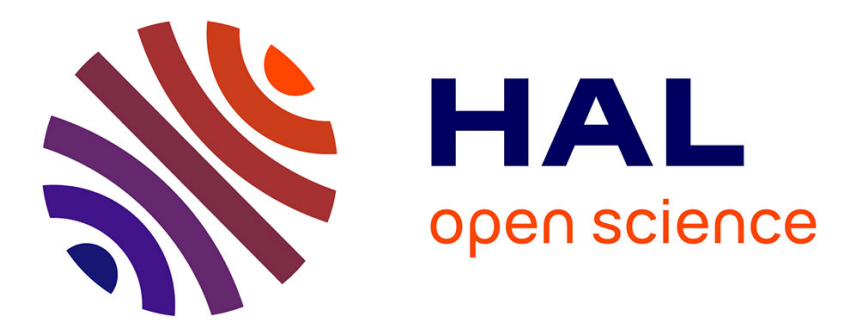

\title{
Trapped light scattering within optical coatings: a multilayer roughness-coupling process
}

\author{
Claude Amra, Myriam Zerrad, Michel Lequime
}

\section{To cite this version:}

Claude Amra, Myriam Zerrad, Michel Lequime. Trapped light scattering within optical coatings: a multilayer roughness-coupling process. Optics Express, 2021, 29 (16), pp.25570-25592. 10.1364/OE.428326 . hal-03323109

\section{HAL Id: hal-03323109 \\ https://hal.science/hal-03323109}

Submitted on 7 Sep 2021

HAL is a multi-disciplinary open access archive for the deposit and dissemination of scientific research documents, whether they are published or not. The documents may come from teaching and research institutions in France or abroad, or from public or private research centers.
L'archive ouverte pluridisciplinaire HAL, est destinée au dépôt et à la diffusion de documents scientifiques de niveau recherche, publiés ou non, émanant des établissements d'enseignement et de recherche français ou étrangers, des laboratoires publics ou privés. 


\title{
Trapped light scattering within optical coatings: a multilayer roughness-coupling process
}

\author{
Claude Amra, ${ }^{*}$ (D) Mrriam Zerrad, (D) and Michel Lequime (D) \\ Aix Marseille University, CNRS, Centrale Marseille, Institut Fresnel, Faculté des Sciences-Campus Saint \\ Jérôme, Avenue Escadrille Normandie-Niemen, 13397 Marseille, France \\ *claude.amra@fresnel.fr
}

\begin{abstract}
Despite numerous works devoted to light scattering in multilayer optics, trapped scattering has not been considered until now. This consists in a roughness-coupling process at each interface of the multilayer, giving rise to electromagnetic modes traveling within the stack. Such a modal scattering component is today necessary for completing the energy balance within high-precision optics including mirrors for gyro-lasers and detection of gravitational waves, where every ppm (part per million) must be accounted for. We show how to calculate this trapped light and compare its order of magnitude with the free space scattering component emerging outside the multilayer.
\end{abstract}

\section{(C) 2021 Optical Society of America under the terms of the OSA Open Access Publishing Agreement}

\section{Introduction}

Light scattering has been extensively studied in multilayer optics [1-8], and today it is possible to predict and measure total losses (integrated in whole space) less than a few ppm ( $1 \mathrm{ppm}=10^{-6}$ of the incident flux) in the whole continuous spectral range $(400 \mathrm{~nm}-1.7 \mu \mathrm{m})[9,10]$. Such accuracy is crucial for applications in high precision optics, including space applications (rejection rates in sensor matrices), mirrors for gyro-lasers (blind zones) and detection of gravitational waves [11-13] (see LISA/LIGO/Virgo projects).

The source of scattering in optical coatings is known to be the roughness at each interface within multilayers [3,4]. This has been largely proved for high-energy deposition techniques (Ion Assisted Deposition, Dual Ion Beam Sputtering, Magnetron Sputtering and others) [14]. Using these techniques, it is known that the substrate roughness replicates at each interface of the stack, hence creating a roughness and scattering threshold. For this reason polishing techniques are key, and substrates must be used with roughnesses less than a fraction of a nanometer in the optical bandwidth of spatial frequencies (typically less than the inverse wavelength) [15-17].

Despite this relevant state of the art, numerous efforts are still devoted to controlling and reducing wide-angle scattering in complex interferential filters [9] (e.g., space multiplexors). The same balance is observed for mirrors for which total losses should not exceed a few ppm (e.g., gravitational waves). Solutions have been proposed to reduce scattering on the basis of destructive interference between the scattering sources [18,19], a process that relies on the generalization of the anti-scattering effect [20] for coatings produced at oblique deposition [3-4]. However these techniques require a unity mutual coherence between all scattering sources; such techniques are extremely sensitive to surface cross-correlation coefficients and cannot be guaranteed in all situations (namely those where the material grain size dominates the replication effect [14]).

Within this context it must be stressed that one effect has not been considered until now in the energy balance of an optical multilayer. This missing term is trapped scattering [21], that is, the amount of light that remains embedded within the stack and cannot emerge into free space. This trapped light results from a coupling process allowed by the interface roughnesses, and propagates in the form of electromagnetic modes guided within the layers. For this reason, we call it a roughness-induced scattering, a process that can be seen by analogy with the well-known grating coupler [22]. The grating coupler is a common technique that has been extensively used 
in experiments where light must penetrate a low thickness waveguide (i.e., when no side coupling is possible). In this case the grating is located at the top of the waveguide and is illuminated by a source in the far field of the incident medium. The grating period must fit the requirements for generating discrete spatial frequencies which attain the modal constants of the guide; the grating shape can be used to optimize the coupling efficiency, though it is mainly driven by the opto-geometrical structure of the guide (including an air gap if necessary).

The process of trapped scattering is much more complex for several reasons. First of all, the deterministic periodic profile of the grating is replaced by a random topography (the roughness) that gives rise to a continuum of spatial frequencies. Secondly, there are several random topographies located at all interfaces in the stack, and each of them must be considered. Also, we have to take account of interference between all modes coupled by the different roughnesses, so that the energy carried by the modes is driven by mutual coherence factors. Eventually the stacks are not reduced to a single layer waveguide, but may include hundreds of layers supporting numerous modes for each polarization.

Hence one key question to answer in this theoretical paper concerns the amount of trapped light in multilayers, and importantly, whether this trapped light is greater or less than the radiative light which emerges outside the stack (in free space in the far field). The result will depend on the statistical nature of roughness and on the associated surface cross-correlation coefficients, as well as on the coating design and on the illumination conditions (incidence, wavelength and polarization).

It should also be stressed that the trapped scattering is involved in the absorption balance of the coating. Indeed, trapped light propagates in the form of guided modes at all polar (azimuthal) directions within multilayers whose imaginary indices most often lie around $10^{-5}$ or $10^{-6}$ [23]; these modes are attenuated over a distance that can exceed one $\mathrm{cm}$, creating an additional "modal" (roughness-induced) absorption that may spread into a region much larger than that of the "classical" absorption. To recall, classical absorption A occurs in the spot size region and its density $d A / d z$ is proportional to the modulus squared of the electromagnetic field [24], with $z$ the altitude within the stack. The result is that modal absorption may dominate the classical absorption, and this information is crucial to avoid any unnecessary optimization of the thin film deposition parameters. An illustration is given in Fig. 1. Note here that we do not consider the decoupling of the trapped light along the rough waveguide, which is a second-order effect.

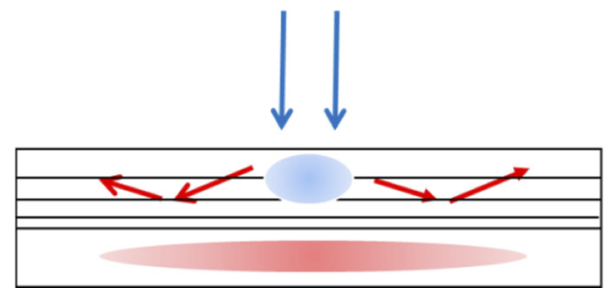

Fig. 1. Schematic diagram of classical and modal absorption. Illumination is given by the blue arrows, with the classical absorption region in blue. The guided modes are in red, and the transverse dimensions of the modal absorption region are illustrated in red.

In Section 2, we recall the key results of a theory we developed [7,25-27] for optical microcavities supporting arbitrary sources in their volume or at their interfaces. The sources are described with magnetic and electric currents. The media can be dielectric or metallic. They are linear, isotropic and non-magnetic. The key result is the formula for the spectral density of the electromagnetic power provided by the cavity. We then show in Section 3 how this theory can be extended to light scattering in multilayers. This extension follows directly from previous works $[6,28]$ devoted to scattering. However, we focus on the scattering power formula, rather 
than on the flux formula currently used in the scattering literature. This is a key point which enables the trapped light problem to be solved. Section 4 is devoted to numerical calculation in typical coatings (mirrors and Fabry-Perot filters) and in more complex stacks (multiple cavity narrow-band filters). In all cases, the trapped light in the coating is quantified for each mode order and polarization, and plotted against spatial frequency and incident wavelength. A detailed comparison is given in regard to free space scattering. Also, a comparison is given for the amount of light trapped under total reflection within the substrate. A conclusion is given in Section 5 . To our knowledge, this is the first time trapped scattering has been quantified within multilayer optics, and we expect that the community will find this work useful.

\section{Electromagnetic power provided by sources within multilayer micro-cavities}

This work first relies on two previous papers $[26,27]$ that we devoted to light emitted by multilayer micro-cavities. The cavities can be all-dielectric or combined with plasmonics. The sources are physical sources located inside the stack, and are described with electric and magnetic currents. These currents can be located at the surfaces or in the bulks within the cavity. The intensity patterns are obtained from Maxwell's equations and the main results (those we need for the trapped scattering) are reviewed in this section. Note that fields and currents do not interact in this work. In what follows we work in the harmonic regime, which means that all quantities $X(\vec{r}, z, \omega)$ are Fourier transforms versus time, with $\omega$ the conjugate Fourier variable of time $(t)$. For the sake of clarity this temporal frequency $\omega$ will be omitted.

We limit ourselves to surface currents, which will describe the situation of roughness scattering (see next Section 3). These currents are tangential and written as:

$$
\vec{J}(\vec{r}, z)=\sum_{i=0}^{p} \vec{J}_{i}(\vec{r}) \delta\left(z-z_{i}\right) \quad \vec{M}(\vec{r}, z)=\sum_{i=0}^{p} \vec{M}_{i}(\vec{r}) \delta\left(z-z_{i}\right),
$$

with $\vec{J}_{i}, \vec{M}_{i}$ the electric and magnetic currents at interfaces located at heights $z_{i}$ in the stack (see Fig. 2), and where $\delta$ is the Dirac distribution. The spatial variable is $\vec{r}=(x, y)$ in a transverse plane parallel to all average surfaces. The layer number of the stack is denoted $p$.

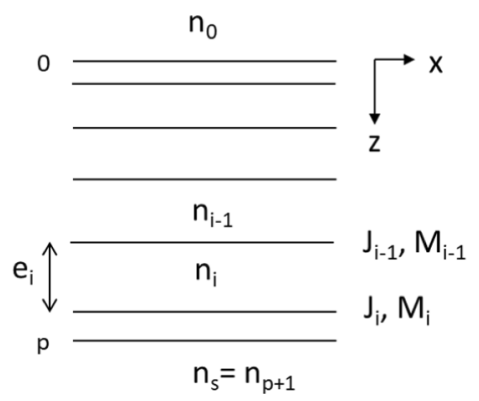

Fig. 2. Geometry of the multilayer cavity, with refractive indices $n_{i}$ and thicknesses $e_{i}$.

The electromagnetic power provided by all sources is classically given as [7]

$$
F=\left(\frac{1}{2}\right) \operatorname{Re}\left\{\int\left(\vec{M}^{*} \cdot \vec{H}-\vec{J} \cdot \vec{E}^{*}\right) d \Omega\right\},
$$

with $d \Omega$ a bulk element, $X^{*}$ the conjugate complex of $X$, and $\vec{E}, \vec{H}$ the electric and magnetic fields. Parseval's theorem applied to Eqs. (1) and (2) in the second Fourier plane leads to [7,25-27]

$$
f(\vec{\sigma})=2 \pi^{2} \sigma \operatorname{Re}\left\{\sum_{i=0}^{p}\left\{\overrightarrow{\hat{M}}_{i}^{*}(\vec{\sigma}) \cdot \overrightarrow{\hat{H}}_{i}(\vec{\sigma})-\overrightarrow{\hat{J}}_{i}(\vec{\sigma}) \cdot \overrightarrow{\hat{E}}_{i}^{*}(\vec{\sigma})\right\}\right\},
$$




\section{Optics EXPRESS}

where $f(\vec{\sigma})$ is the power spectral density, that is, the power provided per unit spatial pulsation $\sigma$ and polar angle $\varphi$

$$
f(\vec{\sigma})=d F / d \sigma d \varphi
$$

with

$$
\vec{\sigma}=\sigma(\cos \varphi, \sin \varphi)=2 \pi \vec{v}
$$

and $\vec{v}$ the spatial frequency.

All currents and fields $\hat{X}(\vec{\sigma})$ in Eq. (3) are tangential and Fourier transforms of $X(\vec{r})$ versus variable, $\vec{r}$, whose conjugate (Fourier) variable is the spatial pulsation $\vec{\sigma}$. The polar angle $\varphi$ gives the azimuthal direction of the emitted wave. At low frequencies that allow light to emerge into the extreme media (substrate and superstrate) the normal angle $\theta$ in the extreme (transparent) media is given by the spatial pulsation (see Fig. 3)

$$
\sigma<k \Rightarrow \sigma=k \sin \theta \quad \text { with } \quad k=2 \pi n / \lambda,
$$

with $\lambda$ the wavelength concerned, and $n$ the refractive index of the surrounding (extreme) medium. Notice also that the fields in Eq. (3) are denoted $X_{i}$ or $X^{\prime}{ }_{i}$ to take account of boundary conditions. Actually both fields are taken at interface $i$ but $X_{i}$ is on the lower side (in medium $i+1$ ) while $X^{\prime}{ }_{i}$ on the upper side (in medium $i$ ).

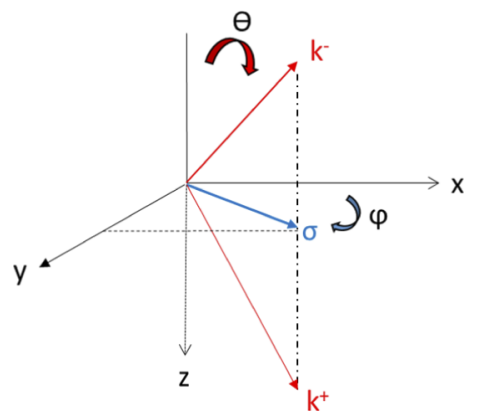

Fig. 3. Relationship between spatial pulsation $\vec{\sigma}$ and wave direction $(\theta, \varphi)$. The wave vector is denoted $\vec{k}$.

To go further we need to express the fields as functions of currents, or conversely. Let us start by keeping the currents. For that, we first notice that each field $X_{i}$ in Eq. (3) is the superposition of fields $X_{j i}$ provided by currents $\left(J_{j}, M_{j}\right)$, that is

$$
\vec{H}_{i}=\sum_{j=0}^{p} \vec{H}_{j i} \quad \vec{E}_{i}^{\prime}=\sum_{j=0}^{p} \vec{E}_{j i}^{\prime} .
$$

Complex admittances $Y_{i}, Y^{\prime}{ }_{i}$ and transfer coefficients $C_{i j}, C^{\prime}{ }_{i j}$ are then introduced [7] to obtain the tangential individual fields $X_{j i}$ in the form

$$
\begin{gathered}
j=i \Rightarrow \vec{H}_{i i}=Y_{i} \vec{z} \wedge \vec{E}_{i i} \\
j>i \Rightarrow \vec{H}_{j i}=Y_{i}^{\prime} \vec{z} \wedge \vec{E}_{j i}^{\prime}=Y_{i}^{\prime} \vec{z} \wedge C_{j i}^{\prime} \vec{E}_{j j}^{\prime} \\
j<i \Rightarrow \vec{H}_{j i}=Y_{i} \vec{z} \wedge \vec{E}_{j i}=Y_{i} \vec{z} \wedge C_{j i} \vec{E}_{j j},
\end{gathered}
$$

where the notation $\wedge$ is used for an outer product of vectors. Equations (8a)-(8c) are necessary since they must be written in regions where the admittances are continuous [7,25-27]. $Y_{i}$ and $Y^{\prime}{ }_{i}$ are calculated by recurrence from the substrate $\left(\tilde{n}_{s}\right)$ and superstrate $\left(\tilde{n}_{0}\right)$ effective indices respectively. 


\section{Optics EXPRESS}

All quantities (admittances and transfer coefficients) depend on the coating design (including the extreme media) and vary with spatial frequency. These formula are valid in transparent or absorbing media, for plane waves $\left(\sigma<k_{0, s}\right)$, guided modes $\left[\max \left(k_{0}, k_{s}\right)<\sigma<\max \left(k_{i}\right)\right]$ and evanescent waves $\left[\sigma>\max \left(k_{i}\right)\right]$, with $\max \left(k_{i}\right)$ the maximum index of the multilayer [7,25-27].

The final result takes the form

$$
f(\vec{\sigma})=2 \pi^{2} \sigma \operatorname{Re}\left(f_{0}+f_{1}\right),
$$

where $f_{0}$ designates the so-called incoherent component. This component is specific to the case where the mutual coherence is zero between the fields emitted from different surface currents, so that these fields do not interfere. It can be expressed in terms of cavity coefficients $\alpha_{i}, \beta_{i}, \gamma_{i}$ as

$$
f_{0}(\vec{\sigma})=\sum_{i=0}^{p}\left\{\alpha_{i}\left|\hat{M}_{i}\right|^{2}+\beta_{i}\left|\hat{J}_{i}\right|^{2}+\gamma_{i} \vec{z} .\left(\hat{M}_{i}^{*} \wedge \hat{J}_{i}\right)\right\},
$$

with

$$
\begin{array}{cl}
\alpha_{i}=-Y_{i} Y_{i}^{\prime} / \Delta Y_{i} & \beta_{i}=1 / \Delta Y_{i}^{*} \\
\gamma_{i}=2 j \operatorname{Im}\left\{Y_{i} / \Delta Y_{i}\right\} & \Delta Y_{i}=Y_{i}-Y_{i}^{\prime} .
\end{array}
$$

Equation (10) shows how the cavity coefficients drive the energy of sources at each spatial frequency, whatever the frequency bandwidth (free space, modal and evanescent). Note that evanescent waves will not contribute to the energy balance of transparent media, due to the imaginary value of $f_{0}$ in this frequency range [7,25-27]. The specificity of the frequency ranges is illustrated in Fig. 4.

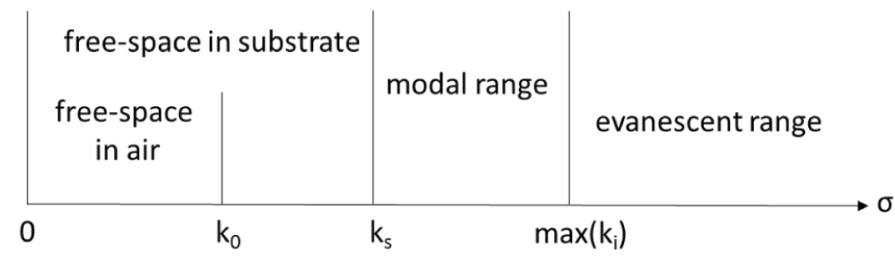

Fig. 4. Schematic diagram of bandwidths associated with plane, modal and evanescent waves, (case $\left.k_{0}<k_{s}\right)$.

Calculation of the correlated component $f_{1}$ is more tedious, and leads to [7,25-27]:

$$
\begin{array}{rl}
f_{1}(\vec{\sigma})=\sum_{\substack{i, j \\
j>i}} f_{i j}+\sum_{\substack{i, j \\
j<i}} f_{i j} & j
\end{array}
$$

with

$$
\begin{aligned}
f_{i j, j>i} & =\frac{C_{j i}^{\prime} Y_{i}^{\prime}}{\Delta Y_{j}}\left[\vec{z} \cdot\left(M_{i}^{*} \wedge J_{j}\right)-Y_{j} M_{i}^{*} \cdot M_{j}\right]+\frac{C_{j i}^{*}}{\Delta \mathrm{Y}_{j}^{*}}\left[J_{i} \cdot J_{j}^{*}-Y_{j}^{*} \vec{z} \cdot\left(M_{j}^{*} \cdot J_{i}\right)\right] \\
f_{i j, j<i} & =\frac{C_{j i} Y_{i}}{\Delta Y_{j}}\left[\vec{z} \cdot\left(M_{i}^{*} \wedge J_{j}\right)-Y_{j}^{\prime} M_{i}^{*} \cdot M_{j}\right]+\frac{C_{j i}^{*}}{\Delta \mathrm{Y}_{j}^{*}}\left[J_{i} \cdot J_{j}^{*}-Y_{j}^{*} \vec{z} \cdot\left(M_{j}^{*} \cdot J_{i}\right)\right] .
\end{aligned}
$$

Equations (14) and (15) emphasize the interaction of all currents, whether they are electric or magnetic. These interactions allow the possibility of light enhancement or inhibition, provided that the mutual coherence of the sources is not zero. 


\section{Optics EXPRESS}

To conclude this section, note that the power density could have also been expressed in terms of the tangential fields, with the result

$$
\begin{gathered}
f_{0}(\sigma)=\sum_{i=0}^{p}\left(Y_{i}\left|E_{i}\right|^{2}-Y_{i}^{\prime}\left|E_{i}^{\prime}\right|^{2}\right) \\
\left.f_{i j, j>i}=C_{j i}^{\prime} Y_{i}^{\prime} E_{j j}^{\prime}\left(E_{i i}^{*}-E_{i i}^{\prime *}\right)+C_{j i}^{\prime *} E_{j j}^{\prime *}\left(Y_{i} E_{i i}-Y_{i}^{\prime} E_{i i}^{\prime}\right)\right] \\
f_{i j, j<i}=C_{j i} Y_{i} E_{j j}\left(E_{i i}^{*}-E_{i i}^{\prime *}\right)+C_{j i}^{\prime *} E_{j j}^{\prime *}\left(Y_{i} E_{i i}-Y_{i}^{\prime} E_{i i}^{\prime}\right) .
\end{gathered}
$$

However, these formulae do not emphasize the cavity coefficients and hence do not allow an analytical discussion of the modes (see Section 3).

\section{Application to roughness-induced scattering in multilayer optics}

When starting with the theory of light scattering in multilayers [2,6,7,28], the boundary conditions are first written for each surface profile. Then both the fields and the surface normal are expanded to first-order under the assumption of low slope and low roughness-to-wavelength ratio, which is appropriate for optical (polished) surfaces and high-precision optics [3,4]. Furthermore, the total field is written as the sum of the scattered field and the (ideal) stationary field that would exist in the absence of roughness. This procedure leads to a system of Maxwell's equations that must satisfy the scattered field. Eventually the system of equations is written with regard to a perfectly planar multilayer supporting electric and magnetic currents at all interfaces. These currents are fictitious in the sense that they result from a mathematical treatment, but the theoretical problem is identical to that of micro-cavities (addressed in the previous section).

Hence we now have to develop the exact form of the scattering currents $\vec{J}_{i}, \vec{M}_{i}$ in order to pass from the micro-cavity problem to the scattering problem. The scattering currents are originally written in the former (spatial) first-Fourier space as [7]

$$
\begin{gathered}
\vec{J}_{i}(\vec{r})=-h_{i}(\vec{r}) \vec{z} \wedge \Delta_{i}\left[\frac{\partial \vec{H}^{0}(\vec{r}, z)}{\partial z}\right] \\
\vec{M}_{i}(\vec{r})=-h_{i}(\vec{r}) \vec{z} \wedge \Delta_{i}\left[\frac{\partial \vec{E}^{0}(\vec{r}, z)}{\partial z}\right]+\operatorname{grad}(h) \wedge \Delta_{i}\left[\vec{E}^{0}(\vec{r}, z)\right],
\end{gathered}
$$

with $\vec{E}^{0}, \vec{H}^{0}$ the (ideal) stationary electromagnetic field, $z=h_{i}(\vec{r})$ the topography of surface $i$, and $\Delta_{i}[\vec{X}]$ the step (discontinuity) in field $\vec{X}$ at surface $i$, with the normal towards $z>0$.

We have already expressed the Fourier transforms of these currents in previous Refs. [6,28]. In the case of illumination by a plane wave of spatial pulsation $\vec{\sigma}_{0}$, they are all proportional to the Fourier transform $\hat{h}_{i}\left(\vec{\sigma}-\vec{\sigma}_{0}\right)$ of the surface profile $i$ with argument $\vec{\sigma}-\vec{\sigma}_{0}$ (a memory effect specific to first-order theory), and to the complex amplitude $\vec{A}^{0}\left(\vec{\sigma}, z_{i}\right)$ of the stationary wave at surface $i$. The result is

$$
\begin{gathered}
\overrightarrow{\hat{J}}_{i}(\vec{\sigma})=j \omega\left(\epsilon_{i+1}-\epsilon_{i}\right) \hat{h}_{i}\left(\vec{\sigma}-\vec{\sigma}_{0}\right) \vec{A}_{t g}^{0}\left(z_{i}\right) \\
\overrightarrow{\hat{M}}_{i}(\vec{\sigma})=-j \omega\left[\frac{\epsilon_{i+1}-\epsilon_{i}}{\epsilon_{i+1}}\right] \hat{h}_{i}\left(\vec{\sigma}-\vec{\sigma}_{0}\right) A_{z, i}^{0}\left(z_{i}\right)(\vec{\sigma} \wedge \vec{z}),
\end{gathered}
$$

with $\epsilon_{i}$ the permittivity of medium $i$, $\omega$ the temporal frequency and $\vec{A}_{t g, i}^{0}, A_{z, i}^{0}$ the tangential and normal components of the stationary wave.

The last step is to introduce Eqs. (21) and (22) into Eqs. (9)-(15) to obtain the power spectral density $f(\vec{\sigma})$ of surface light scattering. Implementation is via 2 computer codes that we developed for light scattering (current formulae) [6,7,28] and for micro-cavities (power formulae) [7,25-27]. 
Numerical results will be given in Section 4. Note that Eqs. (21) and (22) should be further developed to take account of light polarization.

It should be stressed at this stage that all scattering patterns related to low-loss multilayers are calculated in the literature from a flux formulation, and not from a power formulation. So one may wonder why the focus here is on the electromagnetic power, since flux $\Phi$ and power $F$ will be identical in the assumed transparent extreme media ( $F=\Phi+A$, with $A$ the absorption). This key point must be addressed in order to solve the trapped light problem.

The flux formulation $\Phi$ is derived from the Poynting flux of the scattered field through a plane at height $z$ in the extreme media, that is

$$
\Phi=\operatorname{Re}\left\{\int \vec{P}(\vec{r}, z) \cdot \vec{z} d x d y\right\} \quad \text { with } \quad \vec{P}=\left(\frac{1}{2}\right) \vec{E}^{*} \wedge \vec{H},
$$

with a (time) low-pass receiver, this flux is constant if the extreme media are transparent. Using again the admittance formalism and Parseval's theorem, expansion of Eq. (23) in the Fourier space leads to the flux spectral densities by reflection and transmission, that is [7,25-27]

$$
\begin{aligned}
& \frac{d \Phi^{-}}{d \sigma d \varphi}=g^{-}(\sigma)=2 \pi^{2} \sigma \operatorname{Re}\left(\tilde{n}_{0}\right)\left|A_{0}^{-}\right|^{2} \quad \text { in the incident medium } \\
& \frac{d \Phi^{+}}{d \sigma d \varphi}=g^{+}(\sigma)=2 \pi^{2} \sigma \operatorname{Re}\left(\tilde{n}_{s}\right)\left|A_{s}^{+}\right|^{2} \quad \text { in the substrate medium. }
\end{aligned}
$$

All fields in Eqs. (24) and (25) are tangential. The retrograde and progressive scattered fields in the superstrate and substrate are denoted $A_{0}^{-}(\vec{\sigma})$ and $A_{s}^{+}(\vec{\sigma})$ respectively. These flux Eqs. (24) and (25) are well suited to predicting the angular scattering patterns in the extreme media of indices $n_{0}$ (incident medium) and $n_{s}$ (substrate). The total fluxes emerging in free space between $0^{\circ}$ and $90^{\circ}$ by reflection and transmission can be written as

$$
\Phi^{-}=\int_{0}^{k_{0}} g^{-}(\sigma) d \sigma d \varphi \quad \Phi^{+}=\int_{0}^{k_{s}} g^{+}(\widetilde{\sigma}) d \sigma d \varphi .
$$

Observe in the second term in Eq. (26) that the substrate is assumed to be a semi-infinite medium. We also note in Eqs. (24) and (25) the presence of the real part of the effective indices $\tilde{n}$, which removes the contribution of evanescent waves in the energy balance. Indeed we have in the case of transparent extreme media

$$
\sigma>k_{0, s} \Rightarrow \operatorname{Re}\left(\tilde{n}_{0, s}\right)=0
$$

Equation (27) is valid at all high spatial frequencies, including the modal range where the modal constants (or modal frequencies characteristic of guided waves) of the coating may exist [7,25-27]. Recall that this modal range is

$$
\max \left(k_{0}, k_{s}\right)<\sigma<\max \left(k_{i}\right) .
$$

Hence, the flux formulae do not allow the (modal) flux carried by the guided modes to be predicted a priori; using these formulae would give a 0 modal flux. The reason is that the Poynting flux Eq. (23) is calculated through a plane at height $z$, while the modes carry energy perpendicular to these planes. Note also that for an absorbing waveguide, the guided flux is 0 at infinity since the modes are attenuated. The result is that the power $F_{m}$ in that modal bandwidth is totally absorbed $\left(F_{m}=A_{m}\right)$, while the modal flux is $0\left(\Phi_{m}=0\right)$ at infinity. 
On the other hand, the power formalism given by Eqs. (2) and (9) gives the total energy provided by the sources at each spatial frequency, and this energy can be predicted for all directions both in free space and in the modal range. Then the modal power can be written as

$$
F_{m}=\int_{\max \left(k_{0}, k_{s}\right)}^{\max \left(k_{i}\right)} f(\vec{\sigma}) d \sigma d \varphi=2 \pi^{2} \int_{\max \left(k_{0}, k_{s}\right)}^{\max \left(k_{i}\right)} \sigma \operatorname{Re}\left\{f_{0}(\vec{\sigma})+f_{1}(\vec{\sigma})\right\} d \sigma d \varphi .
$$

This quantity can be directly computed once the scattering power density $f(\sigma)$ is known, which is the case at this stage. The major contribution of the integral will be around the real part of the modal constants $\kappa_{q}$ of the coating at which peaks (resonances) occur in the spectral density, with spectral widths proportional to the imaginary parts of these constants, and amplitudes inversely proportional to them [7]. These modal constants $\kappa_{q}$ are given by the complex poles of the reflection factor of the stack [7,25-27], that is

$$
1 / R\left(\kappa_{q}\right)=0
$$

It should be stressed that these reflection poles are identical to those of the power spectral density $f(\sigma)$, that is, to those of the cavity coefficients given in Eqs. (11) and (12) and Eqs. (14) and (15). Indeed Eq. (30) could also be written as $\Delta Y\left(\kappa_{q}, z\right)=0$, a condition which holds for the modes through the entire depth of the stack [7,25-27]. Note also that the functions $R$ or $\Delta Y$ only depend on the modulus of the frequency (not on its direction), so that the modes carry energy at all polar directions $\varphi$ within the stack.

The modal power in Eq. (29) can be calculated in two ways. In the case of absorbing waveguides, the poles $\kappa_{q}$ are complex and cannot be reached by the (real) spatial frequency $\sigma$; hence the spectral density $f(\vec{\sigma})$ shows no singularity so Eq. (29) can be directly computed. On the other hand, for transparent waveguides the poles (when they exist) are real and can be excited by the spatial frequency, so that one has to deal with singularities; the solution is given by the residue theorem [29] which, for a pole located at the boundary, can be written

$$
F_{m}=\int_{\max \left(k_{0}, k_{s}\right)}^{\max \left(k_{i}\right)} f(\vec{\sigma}) d \sigma d \varphi=j \pi \sum_{q} \operatorname{Re} s(f)_{\kappa_{q}} .
$$

Note that singularities must be addressed to avoid any ambiguity, mainly for transparent media. Indeed we know [7,25-27] for transparent media that the cavity coefficients of scattering are purely imaginary at high frequencies (including the modal range). Hence, the scattering power density Eq. (9) is 0 but its integral is not 0, because of its singularities associated with the poles. This is in agreement with the fact that the trapped light is 0 in the absence of modes (or poles). Another way to avoid the ambiguity and summarize this situation is, with $A$ the absorption, to write

$$
\lim _{A \rightarrow 0} F_{m}(A)=\lim _{A \rightarrow 0}\left\{\int_{\max \left(k_{0}, k_{s}\right)}^{\max \left(k_{i}\right)} f(\vec{\sigma}, A) d \sigma d \varphi\right\} \neq \int_{\max \left(k_{0}, k_{s}\right)}^{\max \left(k_{i}\right)}\left\{\lim _{A \rightarrow 0} f(\vec{\sigma}, A)\right\} d \sigma d \varphi .
$$

It should also be noticed that both power and flux densities are imaginary in the modal range for transparent media.

In the next section we carry out numerical calculations for a series of typical coating designs. To this end we consider realistic values for the imaginary indices of the thin films, which are known to be around $10^{-5}$ or $10^{-6}$ in the best situations (actually these values are extremely difficult to measure [23]). Rather than using the residue theorem that involves a path integration 
in the complex plane, we prefer to perform a direct integration on the real axis, as highlighted in Eq. (29). Note that for these low imaginary indices, the resonance peaks are intense so that the major contribution of the integral $F_{m}$ lies around the real parts of the poles.

\section{Numerical calculation for trapped light scattering}

Here we consider common thin film materials that most often constitute the optical coatings for visible-light applications. These are $\mathrm{Nb}_{2} \mathrm{O}_{5}$ (high-index material) and $\mathrm{SiO}_{2}$ (low-index material). The design wavelength for optical coatings will be $600 \mathrm{~nm}$. At this wavelength the real indices are taken as $n_{H}^{\prime}=2.304$ (for $\mathrm{Nb}_{2} \mathrm{O}_{5}$ ) and $n_{L}^{\prime}=1.489$ (for $\mathrm{SiO}_{2}$ ). For simplicity the slight index dispersion of these materials is neglected. The imaginary indices are known to be around $n_{H}^{\prime \prime} \approx 10^{-5}$ for $\mathrm{Nb}_{2} \mathrm{O}_{5}$ and $n_{L}^{\prime \prime} \approx 10^{-6}$ for $\mathrm{SiO}_{2}$. However, the imaginary index for $\mathrm{Nb}_{2} \mathrm{O}_{5}$ will be taken to be around $10^{-4}$, so as to simplify the numerical calculation. Indeed, recall that the peak width is proportional to this quantity, and the peak maximum to its inverse, while we have to integrate density curves for complex filters with numerous narrow peaks in the modal range at all wavelengths. Note also that though the imaginary indices remain difficult to measure, variations around these typical values have negligible impact on the trapped light.

In this section we consider in turn the cases of a mirror and a Fabry-Perot filter. Eventually the trapped light is calculated for a multiple-cavity narrow-band filter. The substrate index is glass with $n_{s}=1.52$. The illumination medium is air, with $n_{0}=1$.

Calculation is carried out for the two polarization modes of the scattered light, denoted $S$ (transverse electric) and $P$ (transverse magnetic). Illumination is at normal incidence $\left(i=0^{\circ}\right)$, which simplifies the out-of-plane calculation (as a function of polar angle $\varphi$ ) of the trapped light for isotropic surfaces. Note that these days almost all surfaces polished on glass are isotropic. With these surfaces the non-polarized scattering pattern has rotational symmetry about the normal of the stack. This is valid for both free space and modal patterns. Indeed, as previously mentioned, the cavity poles are radial since reflection of a (bulk) isotropic stack does not depend on the direction of the spatial frequency, but only on its modulus. Hence numerical calculation of the power spectral densities of scattering will be carried out in the incidence plane $(\varphi=0)$, in which we know that an $S$ (or $P$ ) illumination gives an $S$ (or $P$ ) scattered field (i.e., no cross-polarization at $\phi=0^{\circ}$, as predicted to first-order). Hence the curves will be denoted $S S$ and $P P$ in this plane.

Results will first be plotted in the form of spectral densities $f\left(\sigma, \varphi=0^{\circ}\right)=d F / d \sigma d \varphi$, or integrated over spatial frequency $\sigma$ and plotted in the form of $d F / d \varphi$, that is, the spectral density of modal power per unit polar angle. Polar integration can be performed directly when necessary, relying on the fact that the 4 polarized patterns $\left(S S, S P, P S\right.$ and $P P$ ) vary as $\cos ^{2} \phi$ and $\sin ^{2} \phi$ with the polar angle [7,25-26]. Hence the non-polarized scattering pattern resulting from non-polarized illumination is given by the half sum of these 4 polarized patterns.

Since we are interested in the comparison of trapped (modal) scattering $F_{m}$ to free space (radiative) scattering $F_{\text {rad }}$, we consider the ratio of these quantities (the coupling efficiency), that is

$$
\eta_{m}=F_{m} / F_{\mathrm{rad}}
$$

with

$$
F_{\text {rad }}=\int_{0}^{k_{0}} f(\sigma) d \sigma \quad F_{m}=\int_{k_{s}}^{k_{H}} f(\sigma) d \sigma .
$$

For more detail this ratio can be limited to a particular mode of order $q$, that is, $\eta_{m}^{q}$, with

$$
\eta_{m}=\sum_{q} \eta_{m}^{q}
$$


Recall that the power density peaks are located around the real poles $\kappa_{q}^{0}$ of the cavity calculated in the absence of absorption. Actually the cavity poles $\kappa_{q}$ are complex (otherwise the density peaks would be infinite) but their imaginary values are so small that they can be regarded as similar to the real poles, that is

$$
\operatorname{Real}\left[\kappa_{q}\right] \approx \kappa_{q}^{0} .
$$

We need to keep in mind that flux and power are identical in the free space range for transparent media.

In Eq. (31) we observe that one quantity was not considered, namely

$$
F_{s}=\int_{k_{0}}^{k_{s}} f(\sigma) d \sigma
$$

This corresponds to the quantity of light that remains trapped under total reflection within a substrate of finite thickness (but large with respect to wavelength). Hence we will also consider this key quantity, that is responsible for the side illumination of substrates and which can commonly be seen with the eye. After normalization by the free-space scattering term, it will be denoted as

$$
\eta_{s}=F_{s} / F_{\mathrm{rad}}
$$

Note that, with identical results, this quantity could also be addressed with a modal (interferential and coherent) approach involving the extremely high number of modes within a thick substrate.

The last points to consider are the surface profile parameters. Strictly speaking, we should consider one random roughness at each interface, and numerous cross-correlation coefficients between all surfaces. For the sake of simplicity, the number of parameters can be reduced under the assumption that all surfaces in the stack are uncorrelated with identical roughness spectra $\gamma(v)$. This is the case when optical coatings are deposited onto super-polished surfaces, so that the substrate roughness replication is dominated by the intrinsic roughness brought by the thin film materials $[3,4,20]$. Note that this assumption eliminates the interaction of currents $\left(f_{1}=0\right)$ in Eq. (9). Furthermore, the results will depend on the frequency behavior of the roughness spectrum, and specifically on the correlation length of the surfaces, together with wavelength and incidence. Indeed the greater the slope of the spectrum, the lower the spectrum at high frequencies, and the lower the amount of trapped light at normal illumination. Therefore, for greater generality we will consider a spectrum with slight variations in the range $0<\sigma<\max \left(k_{i}\right)=k_{H}$, though this spectrum corresponds to a realistic roughness value $(1 \mathrm{~nm})$. This will allow the results to be corrected when necessary by introducing a weighting factor describing the decrease in the roughness spectrum.

A 2D Gaussian spectrum can be written as

$$
\gamma(\sigma)=(1 / 4 \pi) \delta^{2} L^{2} \exp \left[\left(-\frac{\sigma L}{2}\right)^{2}\right]
$$

with $\delta$ the roughness and $L$ the correlation length. The condition for this spectrum to be constant at frequencies less than $k_{H}$ requires the correlation length to satisfy

$$
L \ll \lambda / n_{H},
$$

with $\lambda=600 \mathrm{~nm}$ and $L=100 \mathrm{~nm}$, the roughness attenuation at the maximum frequency is around 0.23 , which is not far from the assumption of slight variations. Note also that $100 \mathrm{~nm}$ is a realistic value for a Gaussian correlation length for super-polished surfaces. In what follows we use this Gaussian roughness spectrum with $\delta=1 \mathrm{~nm}$. It should be stressed that though $\delta$ 


\section{Optics EXPRESS}

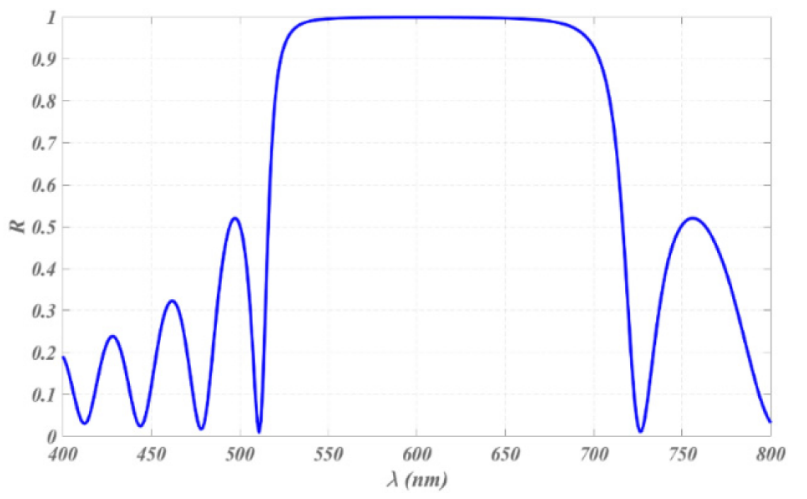

Fig. 5. Optical properties (reflection) of the 17 layer multi-dielectric mirror.

represents a roughness value (root mean square of the topography with 0 mean), strictly speaking this roughness is bandwidth dependent [15-17]. To summarize, since the surface spectrum is quasi-constant and the surface correlation is constant, the $\eta$ ratios are slightly dependent on the topography. As a consequence, the coating design is the key factor that drives the amount of trapped to free-space scattering.

\subsection{Multi-dielectric mirror}

Figure 5 relates to a 17 layer multi-dielectric mirror whose design is classically [30] denoted as Air/ $(H L){ }^{* 8} H /$ Glass. $H$ and $L$ designate high-index $(H)$ and low-index $(L)$ thin film layers with quarter-wave optical thicknesses at the design wavelength $\lambda_{0}=600 \mathrm{~nm}$, that is

$$
(n e)_{H}=(n e)_{L}=\lambda_{0} / 4 \text {. }
$$

The optical properties of this mirror are plotted in Fig. 5 and highlight a reflection bandwidth around the design wavelength $\lambda_{0}$. Illumination is at normal incidence.

In Fig. 6 we have plotted the scattering power density $f(\sigma)$. The illumination wavelength is the design wavelength $\left(\lambda=\lambda_{0}\right)$. Note that the abscissa is the normalized spatial frequency, $v^{*}$, that is:

$$
v^{*}=(\lambda / 2 \pi) \sigma
$$

Such a quantity is dimensionless and can be written as $v^{*}=n \sin \theta$ in the range $v^{*}<n$. Note in Fig. 6 that the free-space, modal and evanescent bandwidths are respectively $B W_{\text {rad }}=\left[0, n_{s}=1.5\right]$, $B W_{m}=\left[n_{s}=1.5, n_{H}=2.3\right]$ and $B W_{e}=\left[n_{H}=2.3, \infty\right]$. The curve in $B W_{\text {rad }}$ can be directly converted in the form of a classical scattering pattern (ARS or BRDF $\cos \theta$ ) as [7]

$$
\operatorname{ARS}(\theta, \varphi)=\operatorname{BRDF}(\theta, \varphi) \cos \theta=k f(\sigma) / \operatorname{tg} \theta
$$

where ARS is used for angle-resolved scattering and BRDF for bidirectional reflectance distribution function. In this $B W_{\text {rad }}$ frequency range, maxima may appear but their amplitudes are bounded because the poles remain far from the real axis regardless of the absorption level. Note that all power quantities take account of light scattered by reflection and transmission simultaneously; there are two sub-ranges in $B W_{\text {rad }}$, that is, a first one $\left(\sigma<k_{0}\right)$ which is a free-space range for both the substrate and superstrate (air), and another $\left(k_{0}<\sigma<k_{s}\right)$ which is a free-space range for the substrate only. Recall that all coupling efficiencies will be normalized with respect to the free-space scattering that can be measured in air, that is, by the integral $F_{\text {rad }}$ of power in the range $\left(\sigma<k_{0}\right)$. 


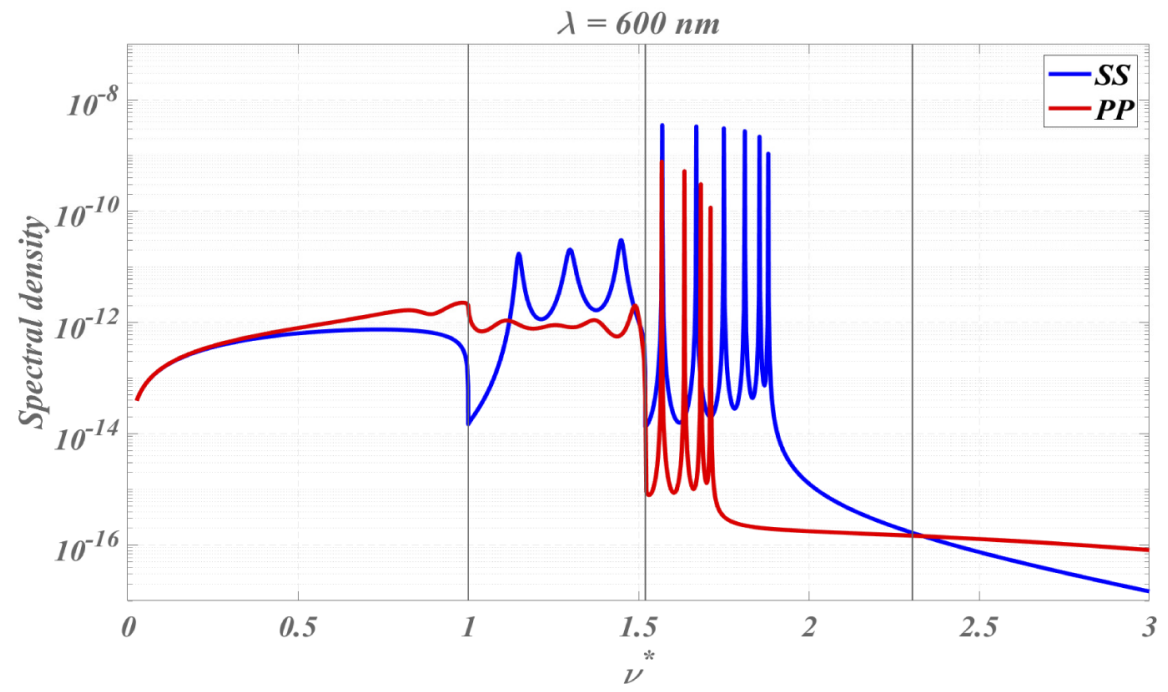

Fig. 6. Scattering power density versus normalized frequency for $S S$ and $P P$ polarization. Case of a 17 layer mirror illuminated at normal incidence at the design wavelength $\lambda=\lambda_{0}=600 \mathrm{~nm}$.

Conversely to the free-space range, the poles can be real in the modal range $B W_{m}$ for transparent stacks, hence sharp maxima can be seen in Fig. 6 for both $S$ and $P$ polarisation modes. As already mentioned, the amplitudes and widths respectively of the peaks tend towards $\infty$ and 0 when absorption vanishes, so that the density pattern would be a Dirac comb in the modal range. Here the peaks are limited in amplitude due to the imaginary indices we used $\left(n_{H}^{\prime \prime} \approx 10^{-4}\right.$ and $n_{L}^{\prime \prime} \approx 10^{-6}$ ). In Fig. 6 several modes can be observed, namely $6 S$-polarized modes and 4 $P$-polarized modes. The zero-order mode is that with the highest normalized frequency. The coupling efficiency $\eta_{m}^{q}$ is given for each mode and polarization in Table 1 . We observe in this table that for TE polarization and depending on the mode order $q$, the trapped light can be greater or less than the free space scattering.

Table 1. Coupling Efficiency $\eta_{m}^{q}$ for Each Mode and Polarization in the Case of a 17 Layer Mirror Illuminated at Normal Incidence at the Design Wavelength $\lambda=\lambda_{0}=600 \mathrm{~nm}$. The Abscissa is the Mode Order $q$.

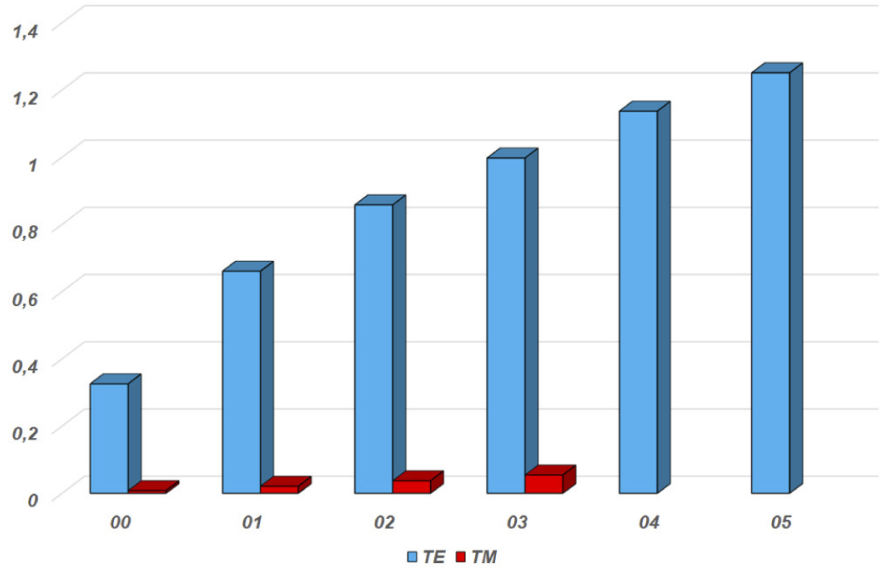


Note in Fig. 6 that the power density is not zero in the evanescent range $B W_{e}$, because of the non-zero values of the imaginary indices. This highlights the weight of evanescent waves in the energy balance of absorbing media, mainly in the absence of modes. However, this weight would be 0 for transparent stacks, that is

$$
v^{*}>n_{H} \Rightarrow f\left(v^{*}, A=0\right)=0 .
$$

In case of a semi-infinite substrate, the range $\left[0, n_{s}\right]$ would be a free-space range in the substrate. With a finite substrate, the free-space range (emerging out of the substrate) is reduced to $\left[0, n_{0}\right]$ while the range $B W_{s}=\left[n_{0}, n_{s}\right]$ is that of total internal reflection within the substrate. Integration of the density in this last $\left(B W_{s}\right)$ range gives the amount of light trapped in the substrate under total reflection (this is different from a modal process).

Since these phenomena are highly chromatic, variations versus both wavelength and spatial frequency are plotted in Fig. 7. The wavelength range is (400 nm; $800 \mathrm{~nm})$, and the power spectral density is given for the half sum of the two polarizations. This figure clearly shows the dispersion path of the poles when the wavelength is scanned. Any vertical line in the modal range allows the number of poles or resonances at each wavelength to be counted. The curves also show how the poles move outside the modal range when the wavelength varies. Note that these poles can no longer be real when they leave the modal range.

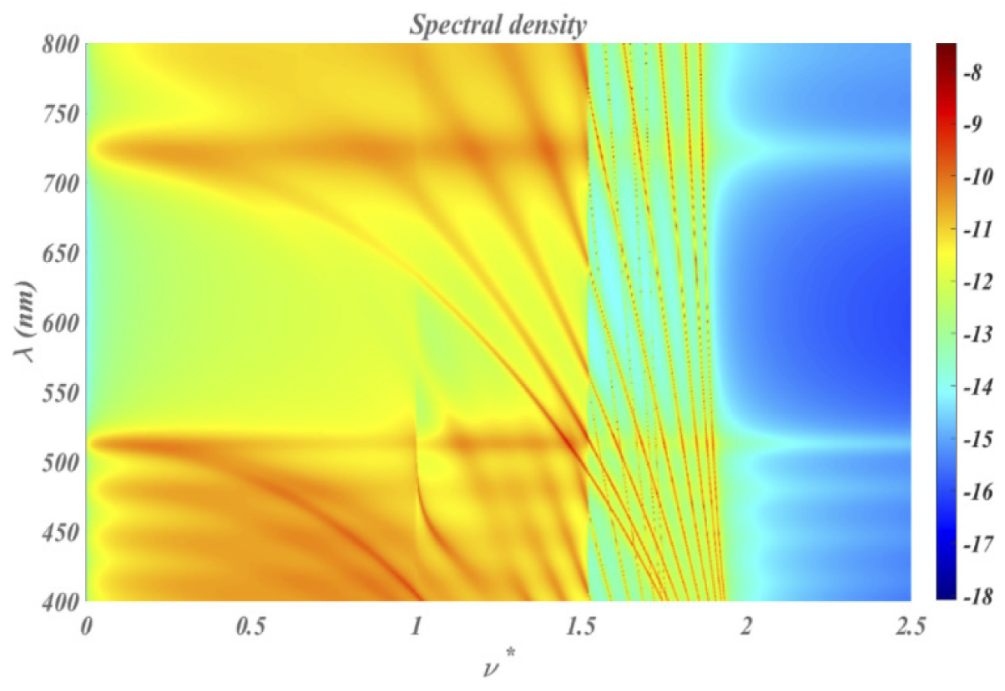

Fig. 7. Variations of the scattering power density versus wavelength and spatial frequency. Case of the 17 layer mirror designed for normal incidence at $600 \mathrm{~nm}$.

All these curves allow the ratios $\eta_{m}(\lambda)$ and $\eta_{s}(\lambda)$ to be calculated as a function of wavelength; this quantifies the amount $\eta_{m}$ of light trapped in the stack in the form of guided waves (i.e., the spectral coupling efficiencies), and the amount $\eta_{s}$ of light trapped under total reflection in a thick substrate of finite thickness. We recall here that these amounts are normalized with respect to the integrated free space scattering $F_{\text {rad }}$ in air. Initially we are interested in the spectral behavior of light trapped $\eta_{m}^{q}(\lambda)$ by each mode, which is shown in Figs. 8 and 9 for TE and TM polarizations. In these figures several modes are present and some of them vanish with increasing wavelength. The first modes which disappear are those of lower spatial frequency (higher-order modes). For more detail, 9 TE modes and 7 TM modes are present at a wavelength of $400 \mathrm{~nm}$, while 4 TE and 3 TM modes remain at $800 \mathrm{~nm}$.

The total amount of trapped light is given in Fig. 10 for both TE and TM polarizations. Two quantities are considered, namely the trapped light in the modal range $\left(\eta_{m}\right)$ which is the energy 


\section{Optics EXPRESS}

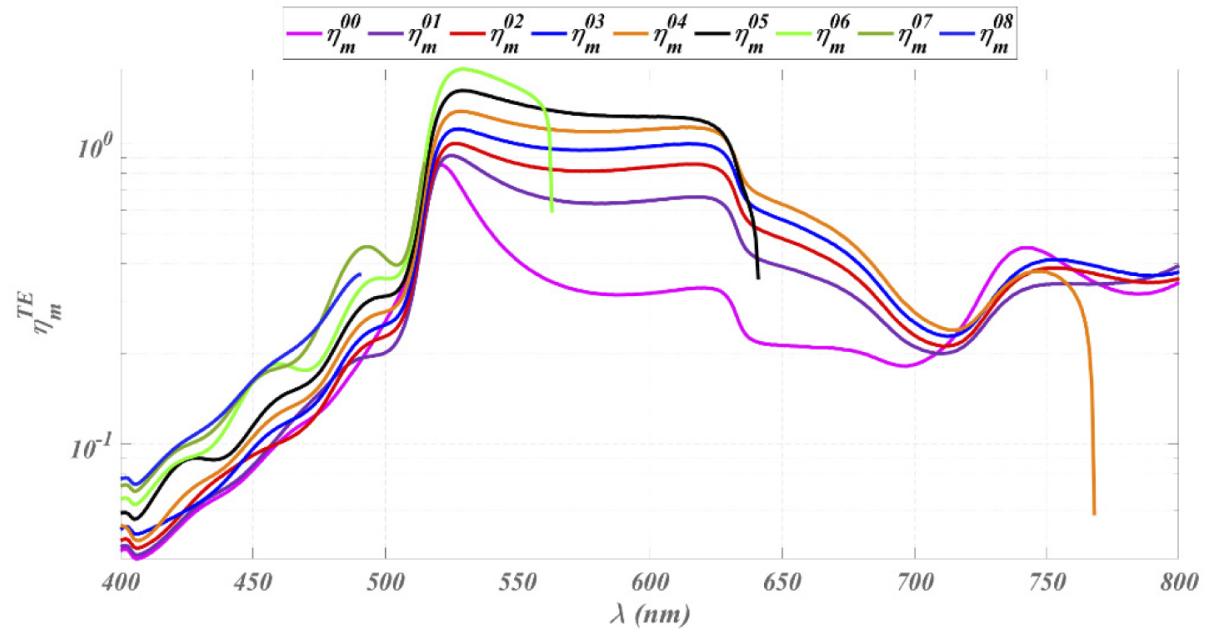

Fig. 8. TE polarization. Amount $\eta_{m}^{q}$ versus wavelength of light trapped by each guided mode of the stack. Normalization is performed with respect to free space scattering in air. Case of a 17 layer mirror with design wavelength $600 \mathrm{~nm}$.

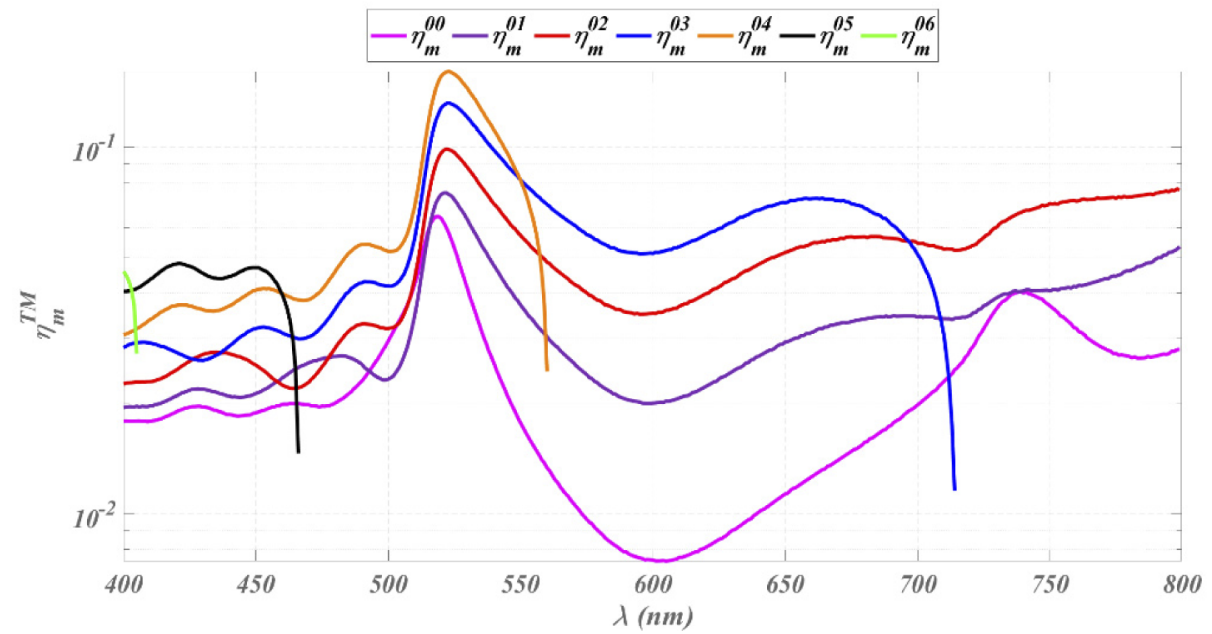

Fig. 9. TM polarization. Amount $\eta_{m}^{q}$ versus wavelength of light trapped by each guided mode of the stack. Normalization is performed with respect to free space scattering in air. Case of a 17 layer mirror with design wavelength $600 \mathrm{~nm}$. 


\section{Optics EXPRESS}

transferred to all modes [see Eq. (35)], and light trapped under total reflection in the substrate $\left(\eta_{s}\right)$ [see Eq. (38)]. We first observe in the whole spectral range that the trapped TE levels are higher than the TM ones, except at short wavelengths. Furthermore these TE levels approach values around 5 (for $\eta_{s}$ ) or 10 (for $\eta_{m}$ ) over a wide spectral range, which means that this TE trapped light dominates the free-space scattering. Modal trapped light $\left(\eta_{m}\right)$ and light trapped under total reflection $\left(\eta_{s}\right)$ have similar orders of magnitude.

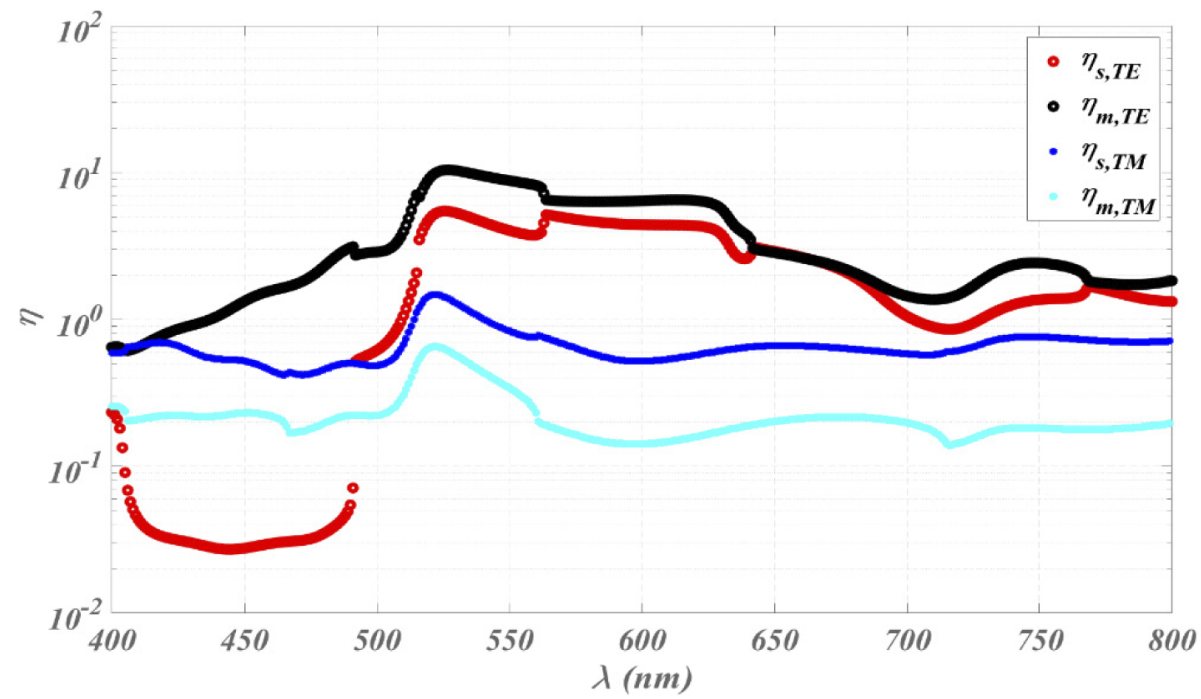

Fig. 10. Total amount versus wavelength of light trapped in the form of guided modes in the stack $\left(\eta_{m}\right)$, or under total reflection $\left(\eta_{s}\right)$ in the substrate. Normalization is performed with respect to free space scattering in air. Case of a 17 layer mirror with design wavelength of $600 \mathrm{~nm}$. Both polarizations are considered.

Note that in the case of an uncoated substrate a single approximate formula can be highlighted for the $\eta_{s}$ ratio. Indeed, following Eq. (9) under the assumption that $f_{0}$ shows negligible frequency variations in the free-space and modal ranges, it is easy to show from Eqs. (34) and (35) that:

$$
\eta_{s} \approx \frac{\int_{k_{0}}^{k_{s}} \sigma d \sigma}{\int_{0}^{k_{0}} \sigma d \sigma}=\left(\frac{n_{s}}{n_{0}}\right)^{2}-1 .
$$

Such a formula shows that the level of trapped scattering in the substrate is similar to that of the free space scattering. However, the assumption of constant $f_{0}$ mainly holds for uncoated substrates.

\subsection{Case of a single-cavity Fabry-Perot filter}

Similar results are given in Figs. 11-16 for a thin film Fabry-Perot filter of design Air/ $M_{4}(6 H) M_{4} /$ Glass, with $M_{4}=H L H L$ a 4-layer mirror and $6 H$ a spacer layer. The design wavelength is $\lambda_{0}=600$ nm. Optical properties of the filter are plotted in Fig. 11, and the power density in Fig. 12. There are $5 S$-polarized and $4 P$-polarized modes at wavelength $600 \mathrm{~nm}$. The $2 \mathrm{D}$ mapping is given in Fig. 13, while the amount of trapped light for each mode versus wavelength is plotted in Figs. 14 and 15 for TE and TM polarizations respectively. At wavelength $400 \mathrm{~nm}, 8 \mathrm{TE}$ modes and $6 \mathrm{TM}$ modes are present, while $4 \mathrm{TE}$ and $3 \mathrm{TM}$ modes remain at wavelength $800 \mathrm{~nm}$. The total amount of light trapped in the coating and in the substrate is plotted in Fig. 16. Conclusions are similar to those of the mirror case. 


\section{Optics EXPRESS}

\subsection{Case of a multiple-cavity filter}

Here we consider a 62 layer multiple-cavity narrow-band filter used for space optics. Multiple cavities are used to obtain a square band-pass with high rejection rates [30]. Optical properties are plotted in Fig. 17 and show 8 decades rejection $(80 \mathrm{~dB})$. The coating is thicker than the previous ones, and this gives rise to a very high number of modes for each polarization, as shown in Fig. 18. The trapped light spectral behavior is given in Figs. 19 and 20 for TE and TM polarizations respectively. The high number of modes creates an intense ripple in the spectral curves which makes them barely readable. The total light trapped in the coating and in the substrate is plotted against wavelength in Fig. 21. Conclusions are similar to those of the previous cases (mirror and single-cavity narrow-band filter).

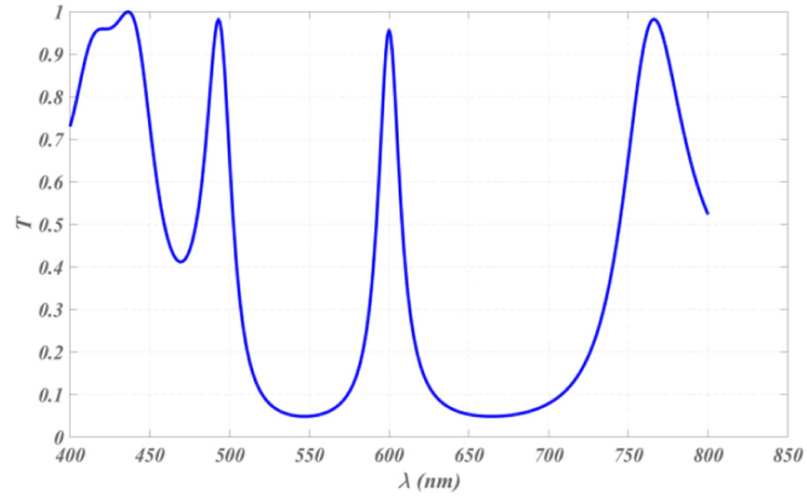

Fig. 11. Optical properties (transmission) of the single-cavity narrow-band filter.

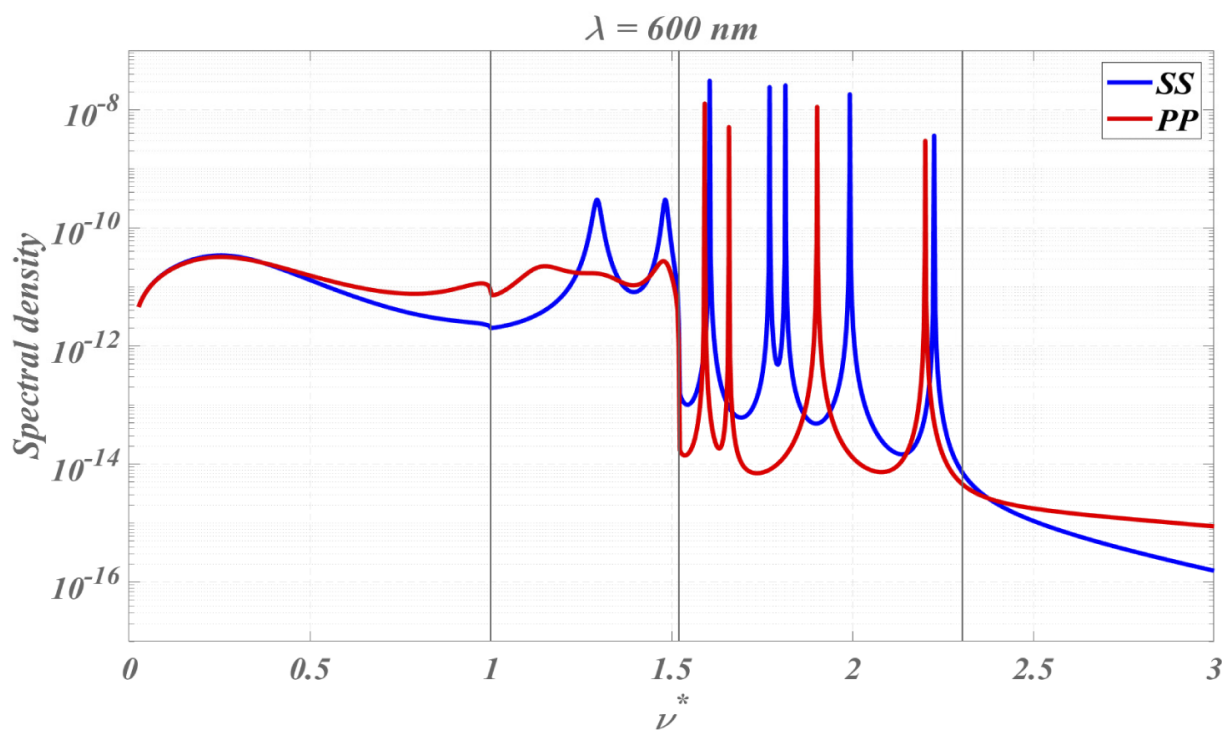

Fig. 12. Scattering power density versus normalized frequency for $S S$ and $P P$ polarizations. Case of a single-cavity Fabry-Perot filter illuminated at normal incidence at the design wavelength $\lambda=\lambda_{0}=600 \mathrm{~nm}$. 


\section{Optics EXPRESS}

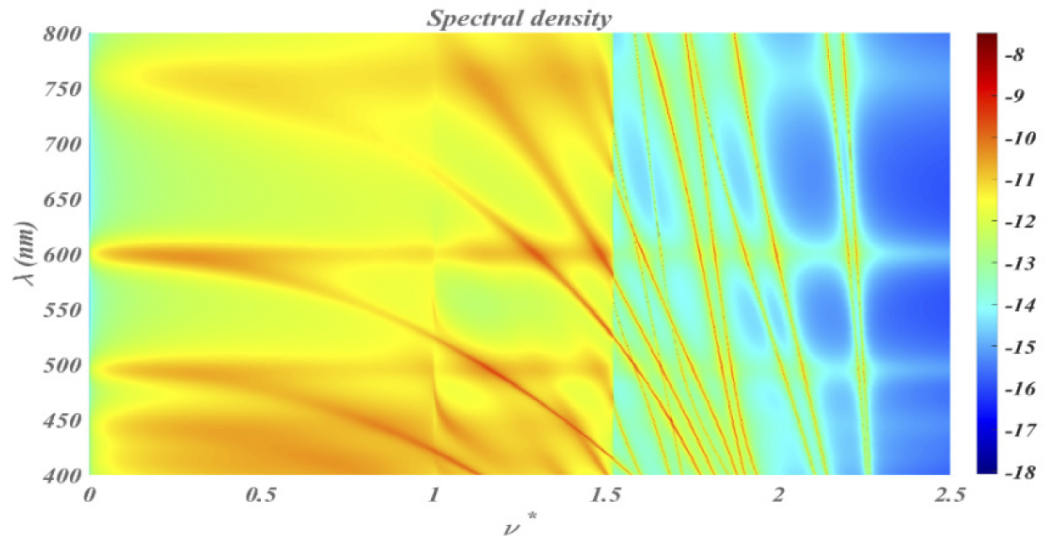

Fig. 13. Variations of scattering power density versus wavelength and spatial frequency. Case of the single-cavity Fabry-Perot filter designed at $600 \mathrm{~nm}$.

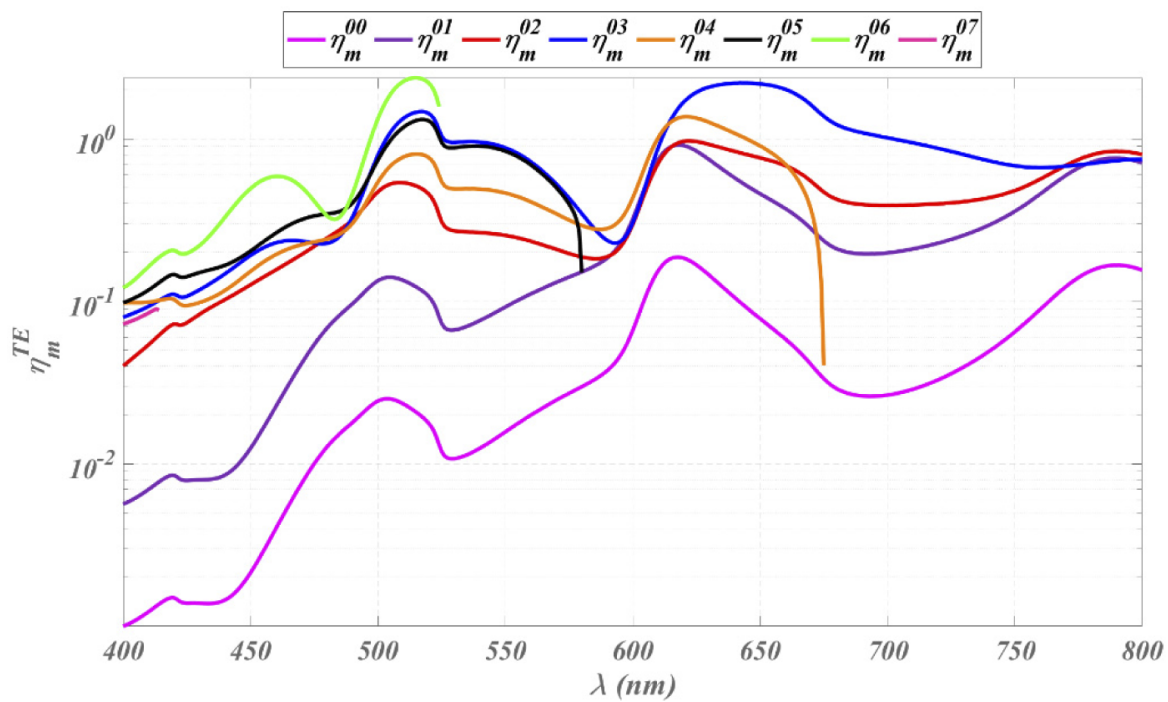

Fig. 14. TE polarization. Amount $\eta_{m}^{q}$ versus wavelength of light trapped by each guided mode of the stack. Normalization is performed with respect to free space scattering in air. Case of the single-cavity Fabry-Perot filter with design wavelength $600 \mathrm{~nm}$. 


\section{Optics EXPRESS}

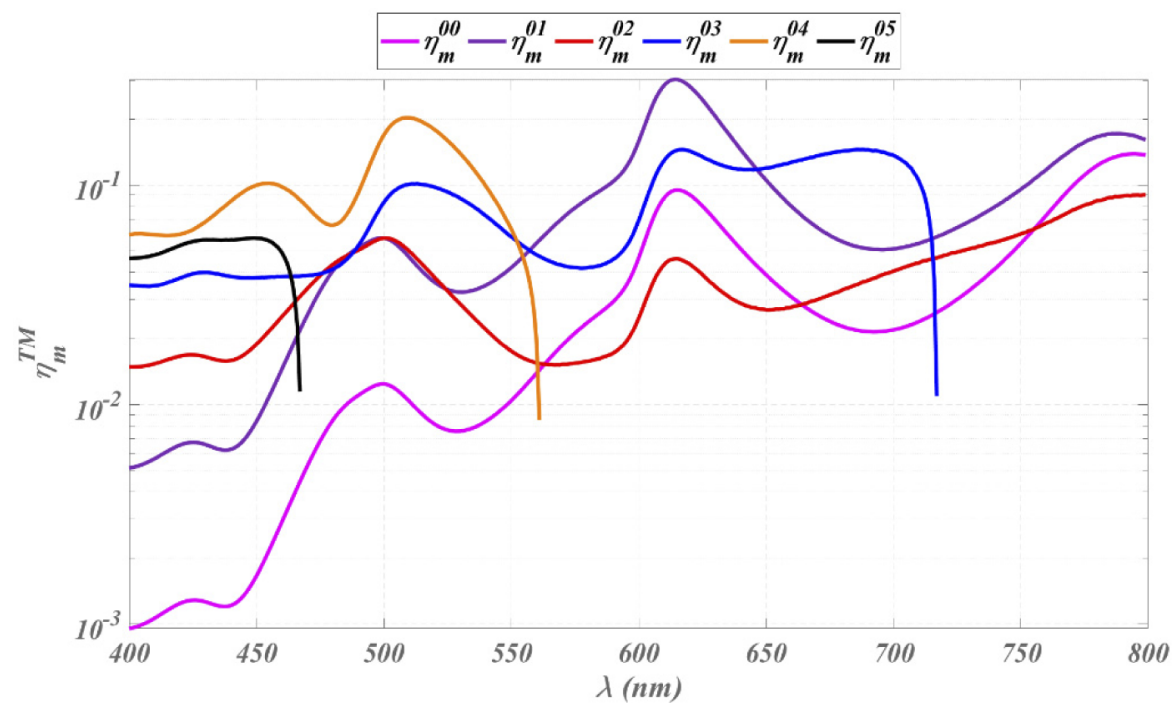

Fig. 15. TM polarization. Amount $\eta_{m}^{q}$ versus wavelength of light trapped by each guided mode of the stack. Normalization is performed with respect to free space scattering in air. Case of the single-cavity Fabry-Perot filter with design wavelength $600 \mathrm{~nm}$.

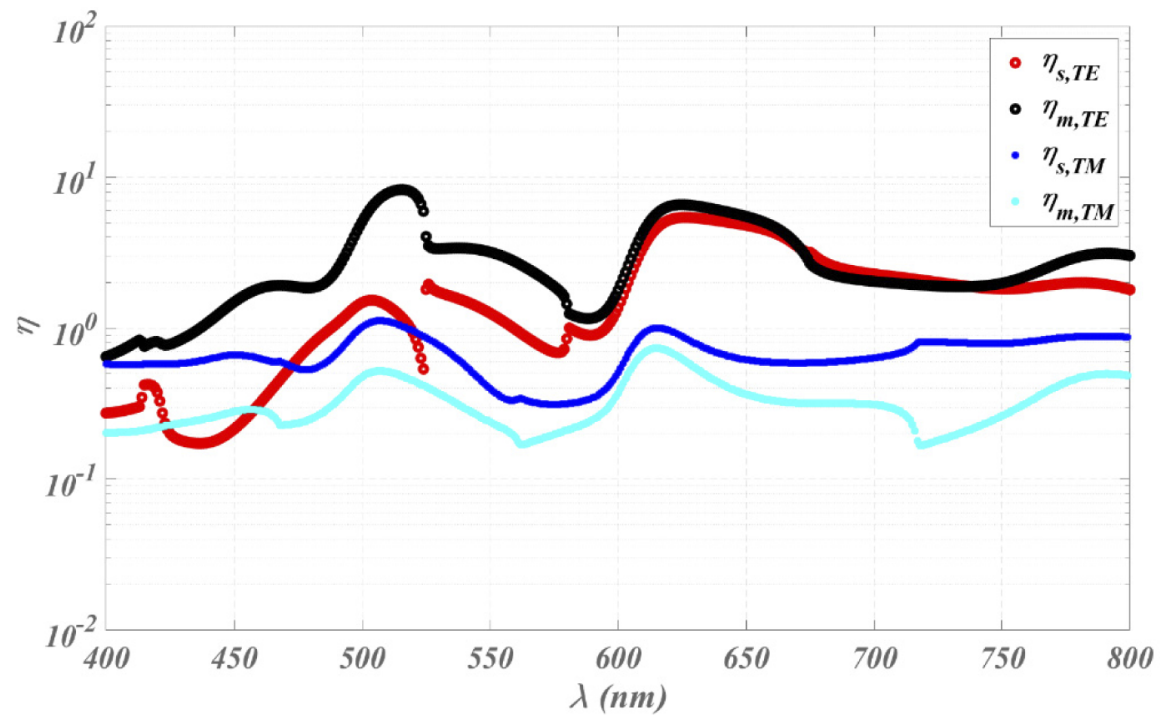

Fig. 16. Total amount versus wavelength of light trapped in the form of guided modes in the stack $\left(\eta_{m}\right)$, or under total reflection $\left(\eta_{s}\right)$ in the substrate. Normalization is performed with respect to free space scattering in air. Case of the single-cavity Fabry-Perot filter with central wavelength $600 \mathrm{~nm}$. Both polarizations are considered. 


\section{Optics EXPRESS}

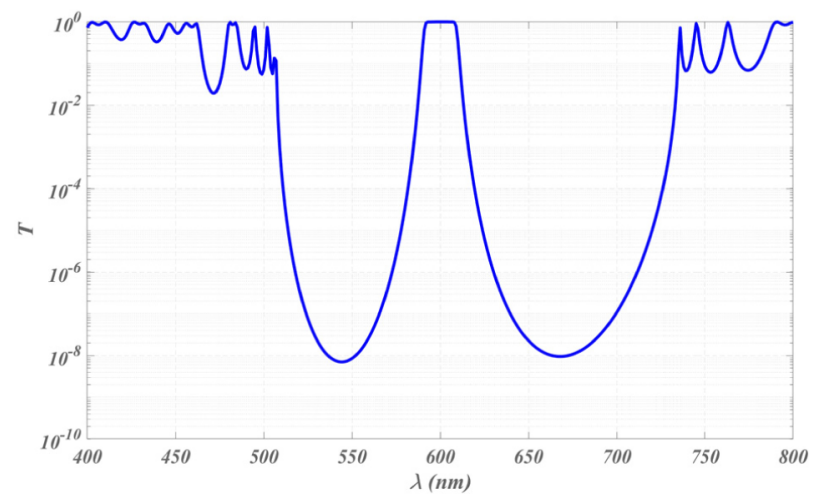

Fig. 17. Optical properties (transmission) of a multiple cavity filter plotted on a logarithmic scale.

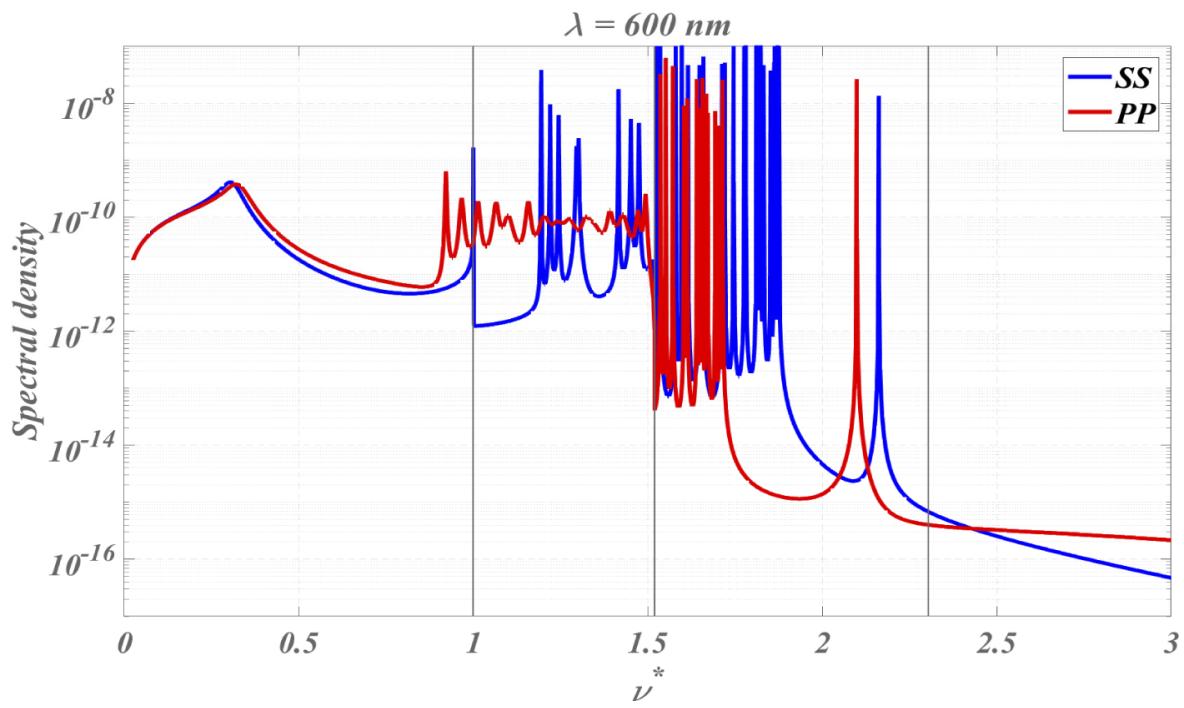

Fig. 18. Power density of scattering versus normalized frequency for $S S$ and $P P$ polarizations. Case of a multiple cavity narrow-band filter designed for normal illumination at wavelength $\lambda=\lambda_{0}=600 \mathrm{~nm}$. 


\section{Optics EXPRESS}

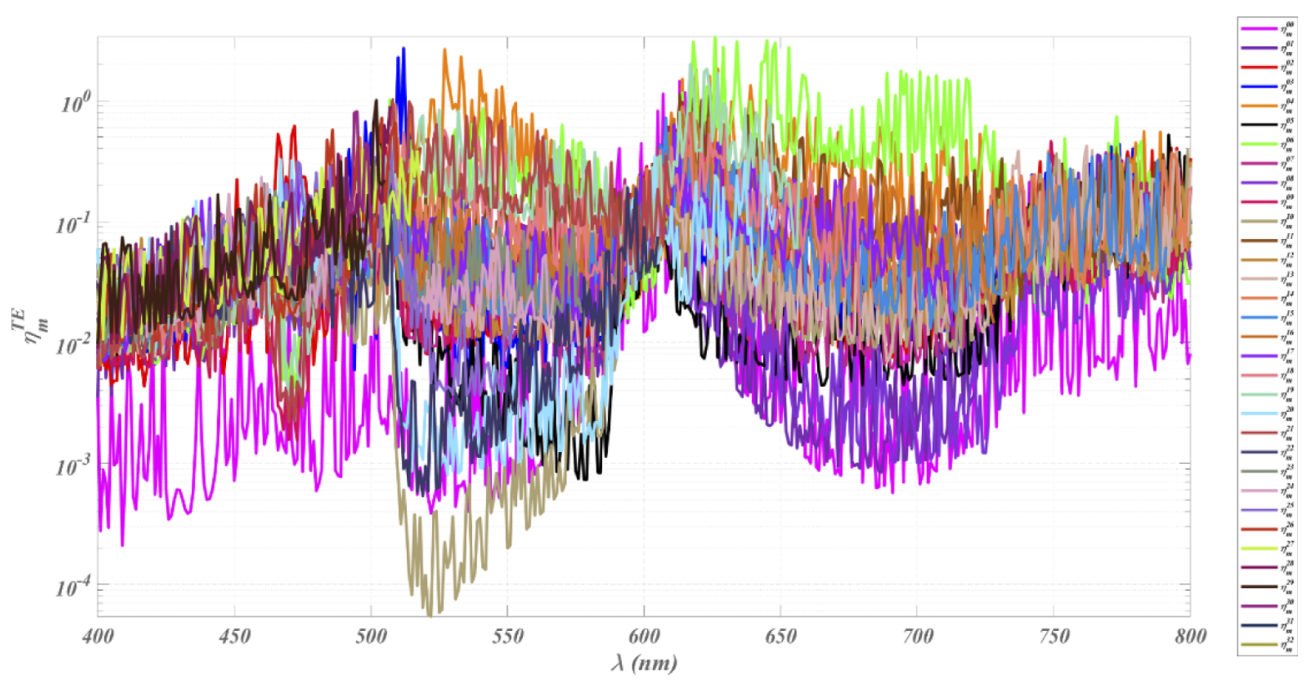

Fig. 19. TE polarization. Amount $\eta_{m}^{q}$ versus wavelength of light trapped by each guided mode of the stack. Normalization is performed with respect to free space scattering in air. Case of the multiple-cavity cavity narrow-band filter with design wavelength $600 \mathrm{~nm}$.

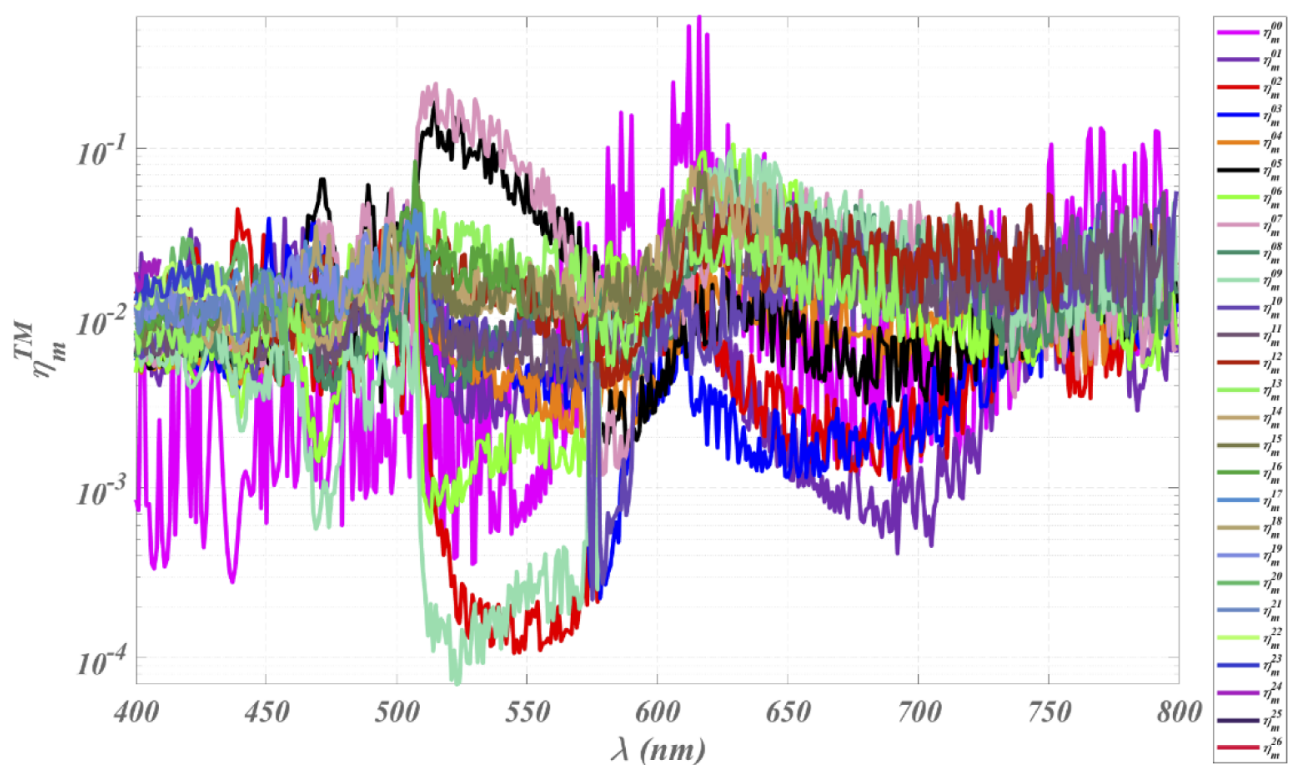

Fig. 20. TM polarization. Amount $\eta_{m}^{q}$ versus wavelength of light trapped by each guided mode of the stack. Normalization is performed with respect to free space scattering in air. Case of the multiple-cavity narrow-band filter with design wavelength $600 \mathrm{~nm}$. 


\section{Optics EXPRESS}

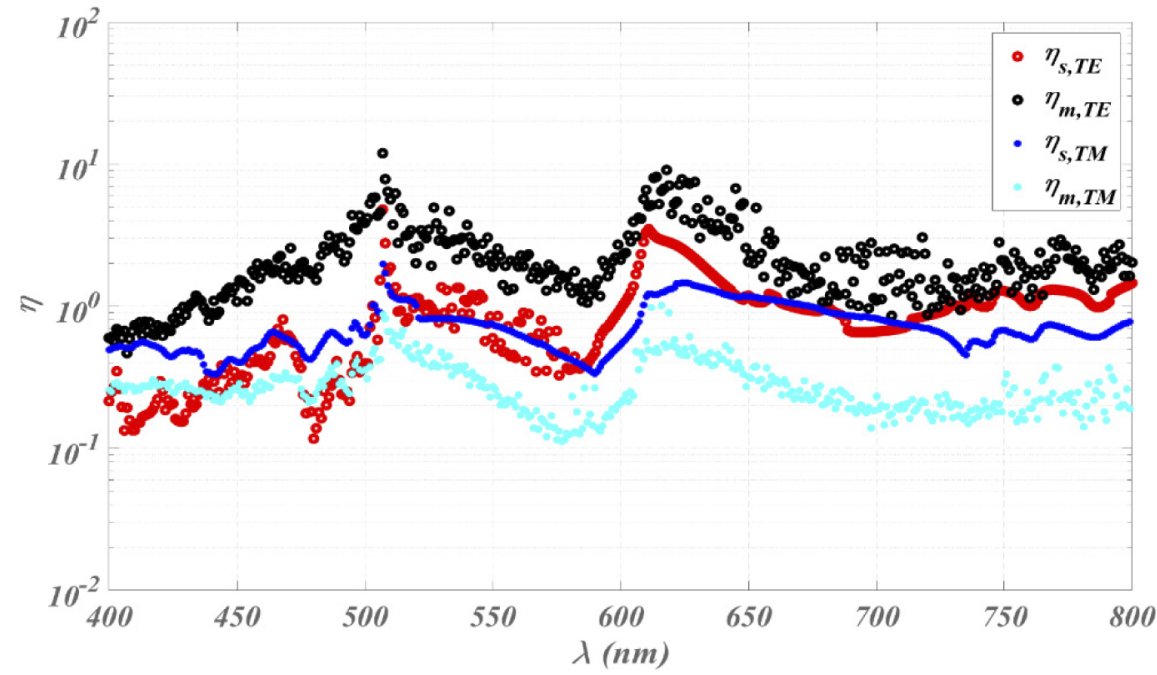

Fig. 21. Total amount versus wavelength of light trapped in the form of guided modes in the stack $\left(\eta_{m}\right)$, or under total reflection $\left(\eta_{s}\right)$ in the substrate. Normalization is performed with respect to free space scattering in air. Case of the single-cavity narrow-band filter with central wavelength $600 \mathrm{~nm}$. Both polarizations are considered.

\subsection{Gratings and localized defects}

It should be stressed that the same formalism allows the extreme sensitivity of the coupling process to be predicted (at least at first-order) when a grating is used. To this end it is sufficient to replace the random surface profile by a periodic one, that is, the Gaussian roughness spectrum by the grating spectrum. Consider first a $1 \mathrm{D}$ grating whose pattern is denoted $h_{0}(x)$. Such a pattern replicates itself in $1(x)$ direction with period $T$, so that the periodic grating profile can be written as

$$
\begin{gathered}
h(x, y)=h_{0}(x, y) *_{x} \sum_{q} \delta(x-q T) \\
\Rightarrow \hat{h}\left(\sigma_{x}, \sigma_{y}\right)=\left(\frac{1}{\mathrm{~T}}\right) \sum_{q} \hat{h}_{0}\left(\frac{2 \pi q}{T}, \sigma_{y}\right) \delta\left(\sigma_{x}-2 \pi q / T\right) .
\end{gathered}
$$

Actually this Dirac comb must be convolved with the illuminated area $S(x, y)$, since the proportionality of the scattered field to $\hat{h}$ only occurs for an illumination plane wave [7]. With $\hat{S}\left(\sigma_{x}, \sigma_{y}\right)$ the Fourier transform of $S(x, y)$, the real spectrum of the surface topography must now be written as

$$
\hat{h}\left(\sigma_{x}, \sigma_{y}\right)=\left(\frac{1}{\mathrm{~T}}\right) \sum_{q} \hat{h}_{0}\left(\frac{2 \pi q}{T}, \sigma_{y}\right) \hat{S}\left(\sigma_{x}-2 \pi q / T\right) .
$$

Recall now that the scattering pattern is the product of the surface spectrum and the cavity coefficients. Hence in the modal range this grating spectrum (not far from a Dirac comb for large illumination areas) must be superimposed onto to the multilayer cavity coefficients (which also tend to another Dirac comb when absorption $A$ vanishes). The coupling efficiency will be negligible unless matching conditions are satisfied in the form $2 \pi q / T \approx \operatorname{Real}\left[\kappa_{q}\right] \approx \kappa_{q}^{0}$ [see Eq. (36)]. Sensitivity increases with the inverse absorption $1 / A$ and with the illuminated area (or the inverse incident divergence). For a 2D grating with 2 periods $T_{x}$ and $T_{y}$, Eq. (47) should be 
replaced by

$$
\hat{h}\left(\sigma_{x}, \sigma_{y}\right)=\left(\frac{1}{T_{x} T_{y}}\right) \sum_{q, p} \hat{h}_{0}\left(\frac{2 \pi q}{T_{x}}, \frac{2 \pi p}{T_{y}}\right) \hat{S}\left(\sigma_{x}-\frac{2 \pi q}{T_{x}}, \sigma_{y}-\frac{2 \pi p}{T_{y}}\right)
$$

and this orthonormal quasi-Dirac comb would intersect the radial quasi-Dirac comb of the multilayer to obtain an optimized coupling efficiency.

In a similar way the coupling process from a single (isolated) localized defect can be predicted. With a circular defect of radius $b$ and amplitude $a$, the defect spectrum is written as

$$
h(x, y)=h(r)=a \operatorname{Circ}(r / b) \Rightarrow \hat{h}(\sigma)=\left(\frac{a}{2 \pi}\right) \int_{0}^{b} r J_{0}(\sigma r) d r,
$$

with $J_{0}$ the 0 -order Bessel function. This last formula shows that small defects in size $\left(b \leq \frac{\lambda}{2 n_{H}}\right)$ will create more (normalized) trapped light in the modal range.

\section{Conclusion}

We have presented a theory for the calculation of trapped light scattering within multilayer optics. To our knowledge, this component of scattering had not been calculated until now, and prediction of its order of magnitude was crucial, especially since no technique is able to measure it directly; indeed this trapped light is absorbed during propagation in the stack, and the propagation length is too short for any local decoupling process to occur without modification to the incident medium. A series of coating designs was considered and it was shown that over a wide range of wavelengths, the trapped scattering in the form of electromagnetic modes within the coating can largely dominate the free space scattering. The same conclusion was drawn for light trapped under total reflection in a substrate of finite thickness. These results prove that trapped scattering can no longer be neglected in any high precision energy balance where each ppm must be eliminated (see for instance the case of mirrors for gyro-lasers or for detection of gravitational waves). Also, it should be kept in mind that this trapped scattering is absorbed during propagation within the stack, which may help in the analysis of the absorption processes (low extinction coefficients, or imaginary indices, may create unexpected absorption levels).

Disclosures. The authors declare no conflicts of interest.

Data availability. No data were generated or analyzed in the presented research.

\section{References}

1. Jean M. Bennett and Lars Mattsson, Introduction to Surface Roughness and Scattering, 2nd ed., (Optical Society of America: 1999)

2. J. M. Elson, J. P. Rahn, and J. M. Bennett, "Relationship of the total integrated scattering from multilayer-coated optics to angle of incidence, polarization, correlation length, and roughness cross-correlation properties," Appl. Opt. 22, 3207-3219 (1983).

3. C. Amra, "Light scattering from multilayer optics. I. Tools of investigation," J. Opt. Soc. Am. A 11, 197-210 (1994).

4. C. Amra, "Light scattering from multilayer optics. II. Application to experiment," J. Opt. Soc. Am. A 11, 211-226 (1994).

5. A. Duparre and S. Kassam, "Relation between light-scattering and the microstructure of optical thin-films," Appl. Opt. 32, 5475-5480 (1993).

6. C. Amra, "First-order vector theory of bulk scattering in optical multilayers," J. Opt. Soc. Am. A 10, 365-374 (1993).

7. C. Amra, M. Lequime, and M. Zerrad, Electromagnetic Optics of Thin-Film Coatings: Light Scattering, Giant Field Enhancement, and Planar Microcavities. (Cambridge University Press, 2020).

8. A. von Finck, T. Herffurth, A. Duparré, S. Schröder, M. Lequime, Myriam Zerrad, S. Liukaityte, C. Amra, S. Achour, M. Chalony, Q. Kuperman, Y. Cornil, A. Bialek, T. Goodman, C. Greenwell, B. Gur, S. Brinkers, G. Otter, A. Vosteen, J. Stover, R. Vink, A. Deep, and D. Doyle, "International round-robin experiment for angle-resolved light scattering measurement," Appl. Opt. 58, 6638 (2019). 


\section{Optics EXPRESS}

9. Marin Fouchier, Myriam Zerrad, Michel Lequime, and Claude Amra, "Wide-range wavelength and angle resolved light scattering measurement setup," Opt. Lett. 45, 2506-2509 (2020).

10. Michel Lequime, Myriam Zerrad, and Claude Amra, "Breakthrough spectrophotometric instrument for the ultra-fine characterization of the spectral transmittance of thin-film optical filters," Opt. Express 26, 34236 (2018).

11. Marcus H. Mendenhall, Robert A. Weller, and Ann F. Whitaker, "Evolution of optical coatings in Earth orbit," Opt. Lett. 16, 1466-1468 (1991).

12. Yi Zhang, Shuyu Yang, Hang Guan, Andy Eu-Jin Lim, Guo-Qiang Lo, Peter Magill, Tom Baehr-Jones, and Michael Hochberg, "Sagnac loop mirror and micro-ring based laser cavity for silicon-on-insulator," Opt. Express 22, 17872-17879 (2014).

13. Simon Zeidler, Tomotada Akutsu, Yasuo Torii, Eiichi Hirose, Yoichi Aso, and Raffaele Flaminio, "Calculation method for light scattering caused by multilayer coated mirrors in gravitational wave detectors," Opt. Express $\mathbf{2 5}$, 4741-4760 (2017).

14. C. Amra, J. H. Apfel, and And E. Pelletier, "Role of interface correlation in light-scattering by a multilayer," Appl. Opt. 31, 3134-3151 (1992).

15. P. Dumas, B. Bouffakhreddine, C. Amra, O. Vatel, E. Andre, R. Galindo, and And F. Salvan, "Quantitative microroughness analysis down to the nanometer-scale," Europhys. Lett. 22, 717-722 (1993).

16. C. Deumie, R. Richier, P. Dumas, and C. Amra, "Multiscale roughness in optical multilayers: atomic force microscopy and light scattering," Appl. Opt. 35, 5583-5594 (1996).

17. M. Zerrad, C. Deumie, M. Lequime, and C. Amra, "An alternative scattering method to characterize surface roughness from transparent substrates,” Opt. Express 15, 9222-9231 (2007).

18. Jinlong Zhang, Shenghuan Fang, Igor V Kozhevnikov, Xinbin Cheng, and Zhanshan Wang, "Interference suppression of light backscattering through oblique deposition of high-reflectivity multilayers: a theoretical analysis," Opt. Express 28, 30626-30643 (2020).

19. Jinlong Zhang, Han Wu, Igor V. Kozhevnikov, Shuaikai Shi, Xinbin Cheng, and Zhanshan Wang, "Interference suppression of light backscattering through oblique deposition of a layered reflecting coating: bi-layer on a substrate," Opt. Express 27, 15262-15282 (2019).

20. C. Amra, G. Albrand, and P. Roche, "Theory and application of antiscattering single layers: antiscattering antireflection coatings," Appl. Opt. 25, 2695-2702 (1986).

21. C. Amra, M. Zerrad, and M. Lequime, "Trapped light scattering within optical multilayers," in Optical Interference Coatings Conference (OIC) 2019, OSA Technical Digest (Optical Society of America, 2019), paper ThD.7.

22. Jun Zou, Yang Zhang, Jinhua Hu, Changhui Wang, Ming Zhang, and Zichun Le, "Grating coupler with reduced back reflection using $\lambda / 4$ offset at its grating sub-teeth,” J. Lightwave Technol. 37, 1195-1199 (2019).

23. Mireille Commandré and Pierre Roche, "Characterization of optical coatings by photothermal deflection," Appl. Opt. 35, 5021-5034 (1996).

24. Bahaa E. A. Saleh and Malvin C. Teich, Fundamentals of Photonics, 3rd ed., (Wiley, 2019)

25. Claude Amra and Sophie Maure, "Electromagnetic power provided by sources within multilayer optics: free-space and modal patterns," J. Opt. Soc. Am. A 14, 3102-3113 (1997).

26. Claude Amra and Sophie Maure, "Mutual coherence and conical pattern of sources optimally excited within multilayer optics," J. Opt. Soc. Am. A 14, 3114-3124 (1997).

27. C. Amra, "Sources within multilayer optics: the energy balance of a planar microcavity," internal publication available on request from the author at the address on the title page

28. C. Amra, C. Grezes-Besset, and L. Bruel, "Comparison of surface and bulk scattering in optical multilayers," Appl. Opt. 32, 5492-5503 (1993).

29. George B. Arfken and Hans J. Weber, "Mathematical Methods for Physicists," Elsevier Academic Press, 6th edition, ISBN 0-12-059876-0

30. H. A. Macleod, "Thin-Film Optical Filters," 4th ed, in Series in Optics and Optoelectronics (CRC Press/Taylor \& Francis, 2010). 